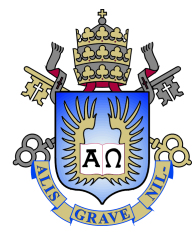

João Gabriel Felizardo Silva Schlittler

\title{
Seleção de carteiras de ativos financeiros via Data-driven Distributionally Robust \\ Optimization
}

Dissertação apresentada ao Programa de Pós-graduação em Matemática da PUC-Rio como requisito parcial para obtenção do grau de Mestre em Matemática.

Orientador : Prof. Marcos Craizer

Coorientador: Prof. Davi Michel Valladão 

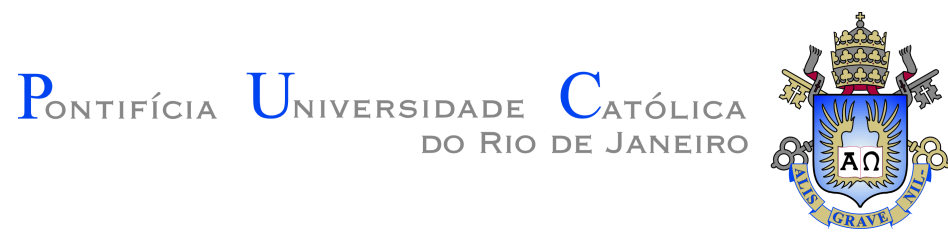

João Gabriel Felizardo Silva Schlittler

\title{
Seleção de carteiras de ativos financeiros via Data-driven Distributionally Robust \\ Optimization
}

Dissertação apresentada como requisito parcial para obtenção do grau de Mestre pelo Programa de Pós-graduação em Matemática da PUC-Rio. Aprovada pela Comissão Examinadora abaixo assinada.

\author{
Prof. Marcos Craizer \\ Orientador \\ Departamento de Matemática - PUC-Rio \\ Prof. Davi Michel Valladão \\ Coorientador \\ Departamento de Engenharia Industrial - PUC-Rio \\ Prof. Alexandre Street de Aguiar \\ Departamento de Engenharia Elétrica - PUC-Rio \\ Prof. Thuener Armando da Silva \\ Departamento de Engenharia Industrial - PUC-Rio \\ Prof. Bruno da Costa Flach \\ IBM Research Brazil - IBM Research \\ Prof. Márcio da Silveira Carvalho \\ Coordenador Setorial do Centro \\ Técnico Científico - PUC-Rio
}


Todos os direitos reservados. É proibida a reprodução total ou parcial do trabalho sem autorização da universidade, do autor e do orientador.

\section{João Gabriel Felizardo Silva Schlittler}

Concluiu a graduação em economia e finanças no Instituto Brasileiro de Mercado de Capitais - Ibmec-RJ

Ficha Catalográfica

Schlittler, João Gabriel

Seleção de carteiras de ativos financeiros via Data-driven Distributionally Robust Optimization / João Gabriel Felizardo Silva Schlittler; orientador: Marcos Craizer; coorientador: Davi Michel Valladão. - 2018.

v., 54 f: il. color. ; $30 \mathrm{~cm}$

Dissertação (mestrado) - Pontifícia Universidade Católica do Rio de Janeiro, Departamento de Matemática.

Inclui bibliografia

1. Matemática - Teses. 2. Otimização robusta, probabilidade e processos estocásticos - Teses. 3. Otimização Distributionally Robust;. 4. Probabilidade;. 5. Otimização de Portfólio;. 6. Otimização sob Ambiguidade;. 7. Otimização sob Incerteza;. 8. Otimização de Portfólio Sob Ambiguidade;. 9. Otimização Data-Driven.. I. Craizer, Marcos. II. Valladao, Davi. III. Pontifícia Universidade Católica do Rio de Janeiro. Departamento de Matemática. IV. Título. 


\section{Agradecimentos}

Uma das muitas coisas que eu aprendi enquanto escrevia minha dissertação, é que nada seria possível sem o apoio de inúmeras pessoas que cruzaram meu caminho, dos quais sou grato por suas contribuições.

Primeiro de tudo, gostaria de agradecer a Deus por me guiar, iluminar e prover a tranquilidade necessária para seguir em frente com os meus objetivos, sem desanimar com as dificuldades.

Agradeço ao meu orientador Marcos Craizer, pois seus conselhos e suporte foram essenciais para atravessar os percursos mais difíceis desta longa jornada. Meu coorientador Davi Valladão e o professor Bruno Fânzeres por suas inúmeras contribuições e por terem sido tão presente na construção deste trabalho, por meio de suas críticas, sugestões e orientações, tendo participado de forma determinante para que ele trabalho acontecesse.

Agradeço à todos os professores do departamento de matemática por todo conhecimento transmitido, e aos amigos de curso, que me acompanharam nas longas jornadas de estudo.

Agradeço minha família que sempre está presente, principalmente meu irmão Luiz André cuja sabedoria e exemplo foram essenciais em todos os meus êxitos. 


\section{Resumo}

Schlittler, João Gabriel; Craizer, Marcos; Valladao, Davi. Seleção de carteiras de ativos financeiros via Data-driven Distributionally Robust Optimization. Rio de Janeiro, 2018. 54p. Dissertação de Mestrado - Departamento de Matemática, Pontifícia Universidade Católica do Rio de Janeiro.

Otimização de portfólio tradicionalmente assume ter conhecimento da distribuição de probabilidade dos retornos ou pelo menos algum dos seus momentos. No entanto, é sabido que a distribuição de probabilidade dos retornos muda com frequência ao longo do tempo, tornando difícil a utilização prática de modelos puramente estatísticos, que confiam indubitavelmente em uma distribuição estimada. Em contrapartida, otimização robusta considera um completo desconhecimento da distribuição dos retornos, e por isto, buscam uma solução ótima para todas as realizações possíveis dentro de um conjunto de incerteza dos retornos. Mais recentemente na literatura, técnicas de distributionally robust optimization permitem lidar com a ambiguidade com relação à distribuição dos retornos. No entanto essas técnicas dependem da construção do conjunto de ambiguidade, ou seja, distribuições de probabilidade a serem consideradas. Neste trabalho, propomos a construção de conjuntos de ambiguidade poliédricos baseado somente em uma amostra de retornos. Nestes conjuntos, as relações entre variáveis são determinadas pelos dados de maneira não paramétrica, sendo assim livre de possíveis erros de especificação de um modelo estocástico. Propomos um algoritmo para construção do conjunto e, dado o conjunto, uma reformulação computacionalmente tratável do problema de otimização de portfólio. Experimentos numéricos mostram que uma melhor performance do modelo em comparação com benchmarks selecionados.

\section{Palavras-chave}

Otimização Distributionally Robust; Probabilidade; Otimização de Portfólio; Otimização sob Ambiguidade; Otimização sob Incerteza; Otimização de Portfólio Sob Ambiguidade; Otimização Data-Driven. 


\section{Abstract}

Schlittler, João Gabriel; Craizer, Marcos (Advisor); Valladao, Davi (Co-Advisor). Portfolio selection via Data-driven Distributionally Robust Optimization. Rio de Janeiro, 2018. 54p. Dissertação de Mestrado - Departamento de Matemática, Pontifícia Universidade Católica do Rio de Janeiro.

Portfolio optimization traditionally assumes knowledge of the probability distribution of returns or at least some of its moments. However is well known that the probability distribution of returns changes over time, making difficult the use of purely statistic models which undoubtedly rely on an estimated distribution. On the other hand robust optimization consider a total lack of knowledge about the distribution of returns and therefore it seeks an optimal solution for all the possible realizations wuthin a set of uncertainties of the returns. More recently the literature shows that distributionally robust optimization techniques allow us to deal with ambiguity regarding the distribution of returns. However these methods depend on the construction of the set of ambiguity, that is, all distribution of probability to be considered. This work proposes the construction of polyhedral ambiguity sets based only on a sample of returns. In those sets, the relations between variables are determined by the data in a non-parametric way, being thus free of possible specification errors of a stochastic model. We propose an algorithm for constructing the ambiguity set, and then a computationally treatable reformulation of the portfolio optimization problem. Numerical experiments show that a better performance of the model compared to selected benchmarks.

\section{Keywords}

Distrubutionally Robust Optimization; Probability; Portfolio Optimization; Optimization Under Ambiguity; Optimization Under Uncertainty; Portfólio Optimization Under Ambiguity; Data-Driven Optimization. 


\section{Sumário}

1 Introdução $\quad 10$

$\begin{array}{lll}1.1 & \text { Revisão Bibliográfica } & 16\end{array}$

$\begin{array}{lll}1.1 .1 & \text { Notação } & 17\end{array}$

2 Base Teórica. $\quad 19$

3 Data-driven Portfolio Optimization Model - DPOM 23

3.1 Apresentação do Modelo 23

3.2 Metodologia para Construção de um Conjunto de Ambiguidade Poliédrico: Uma Abordagem Data-Driven 23

$\begin{array}{lll}3.3 & \text { Reformulação do problema. } & 26\end{array}$

$\begin{array}{lll}3.4 & \text { Tratabilidade } & 28\end{array}$

$\begin{array}{lll}3.5 & \text { Hipóteses } & 30\end{array}$

4 Aplicação do Modelo $\quad 32$

4.1 Estudo de Caso 32

4.2 Experimento Numérico 32

5 Conclusão 46

$\begin{array}{ll}\text { Referências Bibliográficas } & 47\end{array}$

A Apêndice $\quad 52$ 


\section{Lista de figuras}

2.1 Ilustração da condição de aninhamento $(\mathrm{N})$. Dentre os gráficos que mostram diferentes arranjos dos conjuntos $\mathcal{C}_{i}$, somente o primeiro à esquerda respeita a condição $(N)$, enquanto os demais violam. Fonte:[53]

3.1 Família de Fechos Convexos 25

3.2 Conjuntos ascendentes e descendentes do conjunto de incerteza $\mathcal{C}_{2} \quad 29$

3.3 Conjuntos $\overline{\mathcal{C}_{i}}$ gerados com o particionamento do suporte $\mathcal{C}_{0} \quad 29$

3.4 Diagrama de estimação e período implementação do modelo 31

4.1 Máximo Draw Down 34

4.2 Evolução da performance Acumulada (\%) de cada uma das carteiras com 2 ativos contra os respectivos Benchmarks entre 20 de março de 1998 e 29 de março de 2018.

4.3 Relação entre Risco e Retorno das estratégias entre 20 de março de 1998 e 29 de março de 2018.

4.4 Trailing de retornos do modelo Chull $_{10}$ e da carteira $1 / \mathrm{d}$ para vários intervalos (6 meses, 1 aqnos, 2 anos e 3 anos), amostra de 5040 observações diários entre os dias 20 de março de 1998 e 29 de março de 2018

4.5 Alocação percentual em relação a riqueza total das carteiras

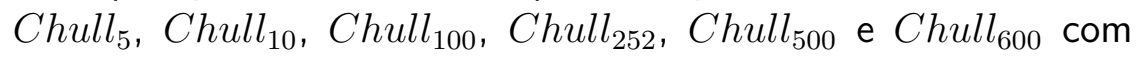
2 ativos, ao longo do tempo, entre os dias 20 de março 1998 e 29 de março de 2018.

4.6 Gráfico de dispersão do retorno dos ativos arriscados, seus respectivos conjuntos de incerteza e intervalos de confiança: Portfólio Industry com 2 ativos em diferentes condições de mercado.

4.7 Gráfico de dispersão do retorno da carteira de 2 ativos, formada com os índices SP500 e Down Jones, seus respectivos conjuntos de incerteza. 


\section{Lista de tabelas}

4.1 Tabela comparativa da estratégia $\mathrm{Chull}_{N}$, com 2 ativos, para diferentes especificações. Amostra entre 20/03/1998 e 29/03/2018, totalizando 5040 observações.

4.2 Proporção (\%) dos retornos positivos e negativos observados de cada estratégia com 2 ativos, em diferentes intervalos de tempo do traling em relação a amostra que 20 de março de 1998 e 29 de março de 2018.

4.3 Tabela com a proporção de dias que as estratégias geraram rendimentos positivos e a proporção de dias que superaram as carteira $1 / d$ e o índice SP500 em relação ao retorno gerado.

4.4 Tabela comparativa com a proporção de dias que houve retorno positivo carteiras com 2, 5, 10, 17 e 30 ativos.

4.5 Tabela comparativa da volatilidade dos retornos diários das carteiras com 2, 5, 10, 17 e 30 ativos.

A.1 Tabelas com o retorno diário médio das carteiras com 2, 5, 10, 17 e 30 ativos.

A.2 Tabelas com a proporção de dias em que cada uma das carteiras Chull com 2, 5, 10, 17 e 30 ativos ganhou da carteira 1/d.

A.3 Tabelas de performance das carteiras elaboradas com o DPOM com 5 e 10 ativos.

A.4 Tabelas de performance das carteiras elaboradas com o DPOM com 17 e 30 ativos. 


\section{Introdução}

Durante décadas, o universo de estratégias de investimento foi amplamente dividido em dois campos: estratégias fundamentalistas e quantitativas (Quants). Os primeiros geralmente tomam decisões de investimento com base em inúmeras métricas, como as demonstrações de lucros, setor de atuação, análise macroeconômica, ou seja, nos fundamentos da empresa. Já os quants estavam menos preocupados com estes fundamentos e mais interessados em saber o que os dados históricos poderiam dizer sobre o movimento futuro dos preços dos ativos. Neste caso, para uma estratégia ser bem-sucedida, ela precisa ser capaz de captar as tendências dos preços dos ativos em seus estágios iniciais, à medida que os computadores executam constantemente cenários para localizar ineficiências antes que outros o façam. Apesar dos modelos serem capazes de analisar um grande grupo de investimentos simultaneamente, onde analistas tradicionais podem olhar apenas alguns de cada vez, sua eficiência depende essencialmente de dois componentes: a qualidade dos dados utilizados e a base teórica do próprio modelo.

Com a evolução tecnológica, testemunhamos uma explosão na quantidade disponível de dados que são rotineiramente coletados em tempo real em diversos setores da economia, o que facilitou a utilização de um número crescente de técnicas quantitativas, as quais, se tornaram muito difundida em diversos setores, em especial na administração de investimento. Primeiramente foi aplicada na gestão do risco proveniente de diferentes fontes, mas nos dias de hoje, os modelos quantitativos são considerados imprescindíveis em todas as principais áreas de gestão de investimentos, onde possui uma vasta e crescente lista de aplicações, como modelos de precificação de opções, otimização de portfólio para alocação de ativos, algoritmos autônomos para execução ordens, et cetera.

Toda esta evolução teve como origem o artigo Portfolio Selection publicado por Harry Markowitz [35], que foi um grande avanço em direção a gestão quantitativa de carteiras, fornecendo as bases da teoria moderna de portfólio como um problema matemático. Seu modelo, que é conhecido como otimização de média-variância, fornece uma estrutura para construir portfólios, tendo como base o valor esperado dos investimentos e o nível de aversão ao risco do 
investidor, que é quantificado pela variância. A grande contribuição desta teoria foi a mudança do foco da análise de investimento, que na visão do modelo, tinha como essência a seleção individual de ativos em direção ao conceito da diversificação e o impacto de cada ativo nas características de risco e retorno do portfólio.

Atualmente é de conhecimento comum que os retornos dos ativos não são normalmente distribuídos e simétricos, logo, apenas o valor esperado e a estrutura de covariância não descrevem completamente a distribuição conjunta do retorno dos ativos, consequentemente muitos riscos e cenários indesejados por gestores de portfólios não podem ser capturados apenas com o valor esperado e a variância. Esta questão foi o estopim para que houvesse, nas últimas décadas, uma "corrida" para o desenvolvimento de modelos que melhor mensurassem o risco, com o intuito de aperfeiçoar sua administração em portfólios financeiros. Dentre as medidas desenvolvidas, temos as medidas de risco de downside, que possuem o objetivo de maximizar a probabilidade de um portfólio gerar retornos acima de algum nível aceitável, também referido como nível benchmark.

Em seu livro original [34], Markowitz propôs o uso da semivariância com o intuito de corrigir o fato que a variância penaliza igualmente retornos positivos e negativos. A semivariância [33], por exemplo foi uma tentativa de considerar a assimetria das distribuições e caracterizar o risco apenas como os retornos negativos. O Value ate Risk (VaR) [27], que é provavelmente a medida de risco mais conhecida, está relacionado com os percentis de distribuições de perdas, quantifica o valor da perda financeira máxima de um investimento dado um período de tempo e nível de significância. Apesar do amplo uso prático, havia uma falta de estudos que definissem quais características uma medida de risco desejável precisaria ter [41]. Neste sentido, surgiu toda uma corrente na literatura que discute, propõe e critica as propriedades teóricas que determinada medida de risco deve cumprir. Com base nesta discussão teórica, o uso indiscriminado do VaR passou a sofrer fortes crítica, devido a suas deficiências, e com isto, [1] apresenta um conjunto de propriedades que uma medida de risco deve possuir, definindo as medidas coerentes de risco.

O Conditional Value at Risk (CVaR) [40], [49] que é uma medida coerente de risco, avalia a probabilidade, dado um certo nível de confiança, de haver uma perda que exceda o VaR, passou a ser defendido como medida de risco a ser utilizada. Matematicamente falando, o CVaR é derivado da média condicional entre Value at Risk e as perdas que o excedem.

Diversos modelos de otimização apresentam parâmetros, que apesar de não serem conhecidos ao certo, são utilizados como estimativas precisas, de 
forma a possibilitar a resolução do problema. No entanto, na presença de erros de estimação, a solução encontrada pode diferir substancialmente do real solução ótima. Os erros de estimação na estratégia de portfólio ótimo pode produzir pesos extremos para cada ativo, gerando grandes flutuações através do tempo como também performances ruins fora da amostra. Por exemplo [36] documenta a necessidade de uma longa série histórica de retorno para obter uma estimativa precisa do retorno esperado. Em adição, [26] relata que a estimativa da matriz de variância-covariância é mal comportada. [38] mostra que a contribuição relativa do erro da matriz de covariância depende principalmente do índice de Sharpe da carteira de mercado e da frequência de amostragem dos dados históricos. Por esta razão, muitos autores propuseram extensões do modelo média-variância [35] para reduzir erros de estimação, com o intuito de melhorar a performance do modelo. Para maiores detalhes, ler [17], $[32]$.

Otimização robusta (RO) é uma área de estudo que visa tornar o modelo matemático factível em relação às restrições do problema para um determinado conjunto definido pela incerteza nos parâmetros, através da resolução de uma contraparte robusta, a qual é dada através da seguinte restrição semi-infinita (1.1). Dessa forma, há uma redução da dependência do resultado do modelo aos parâmetros incertos.

$$
v(\mathbf{x}, \mathbf{r}) \geq \gamma \quad \forall \mathbf{r} \in \mathcal{C}
$$

ou equivalente

$$
\inf _{\mathbf{r} \in \mathcal{C}} v(\mathbf{x}, \mathbf{r}) \geq \gamma
$$

onde $\mathcal{C} \subset \mathbb{R}^{d}$ é o conjunto de incerteza dos retornos, $\mathbf{r} \in \mathbb{R}^{d}$ é o vetor dos retornos, $\mathbf{x} \in \mathbb{R}^{d}$ é a variável de decisão, $v: \mathbb{R}^{d} \times \mathbb{R}^{d} \rightarrow \mathbb{R}$ a função objetivo, convexa. A restrição (1.1) torna robusta a solução do problema de otimização, ao requerer que a alocação $\mathbf{x}$ seja viável para todas as realizações do retornos $\mathbf{r} \in \mathcal{C}$.

Os primeiros trabalhos deste tipo datam da década de 50 , onde se dedicavam na análise do pior caso e modelos maxmin (ou minmax), porém na década de 70, ganhou força com o trabalho de [47], que considerou um modelo determinístico de otimização linear viável para todos os elementos de um conjunto convexo. Atualmente existem inúmeros trabalhos sobre otimização robusta, como [4], [5], [6], [9], [19], [20].

O conceito de robustez está intrinsecamente atrelado a definição de um conjunto de incerteza $\mathcal{C}$, cuja geometria é fundamental para a tratabilidade 
computacional do problema, e com isto, ser possível elaborarmos um problema robusto (contraparte robusta), através de reformulações no problema original, que gere soluções imunes a erros de estimação dos dados de entrada, tal que cada solução no novo problema seja viável para todas realizações do modelo original.

O trabalho [47] apresentou a ideia de utilizar a norma supremum para representar o conjunto de possíveis valores para os parâmetros incertos. Neste modelo é utilizado o nível máximo de proteção possível contra o pior caso e assume-se que todos os parâmetros incertos realizarão simultaneamente o pior resultado possível. A vantagem é que o método possui uma implementação muito simples, porém ele gera um elevado grau de conservadorismo em suas soluções. Para reduzir o conservadorismo desta representação, [6] propõe uma estrutura geométrica elipsoidal para o conjunto de incerteza. A intuição deste modelo se refere ao conceito de que a probabilidades de todos os parâmetros assumirem ao mesmo tempo seus piores casos é muito baixa. A vantagem desta proposta é a possibilidade de controlar o nível de conservadorismo através do diâmetro do conjunto, a desvantagem está no aumento da complexidade do problema. Na mesma linha de redução de conservadorismo, [9] propôs uma representação poliédrica de fácil implementação, onde a ideia é ajustar a robustez da solução em relação às preferências individuais e atitudes em relação ao risco do tomador de decisão, através de um parâmetro ajustável $\Gamma$.

Apesar da simplicidade ao caracterizar incerteza através de conjuntos, otimização robusta tem sido muito bem sucedida ao oferecer modelos computacionalmente tratáveis para uma ampla variedade de problemas complexos. No entanto, nota-se que tipicamente as estruturas dos problemas de RO não requerem nenhuma informação sobre a distribuição dos parâmetros do modelo, e por isto, não exploram tais informações distribucionais que possam estar disponíveis. Consequentemente, nestes casos, RO podem propor soluções excessivamente conservadoras. Em uma linha diferente, otimização estocástica explicitamente conta com informações sobre as distribuição de probabilidade dos parâmetros, e as modela através de restrições esperança, na forma:

$$
\mathbb{E}_{\mathbb{P}^{0}}[v(\mathbf{x}, \tilde{\mathbf{r}})] \geq \gamma
$$

onde $\tilde{\mathbf{r}} \in \mathbb{R}^{d}$ é o vetor dos retornos aleatório, $\mathbf{x} \in \mathbb{R}^{d}$ é a variável de decisão, $v: \mathbb{R}^{d} \times \mathbb{R}^{d} \rightarrow \mathbb{R}$ a função objetivo, convexa e a esperança, que é tomada com respeito a distribuição $\mathbb{P}^{0}$ de $\tilde{\mathbf{r}}$. Contudo, as principais críticas ao modelo de otimização estocástica são, que estes problemas podem demandar um alto custo computacional, como também, na prática, raramente as informações 
sobre a distribuição estão disponíveis. Além disto, há o fato de que a própria distribuição de probabilidade está sujeita a incerteza, e com isto, tais modelos podem levar a soluções enganosamente ótimas.

A incerteza em relação a distribuição levou muitos autores investigarem portfólios sob informação ambígua em relação aos retornos dos ativos. Apesar do grande esforço necessário para lidar com erros de estimativa, estratégias de portfólio são incapazes de dominar consistentemente a carteira $1 / \mathrm{N}^{1}$. O trabalho [39] mostrou que a estratégia de investimento uniforme é racional para investidores que lidam com um alto grau de ambiguidade em relação a distribuição de perda, para varias classes de medidas de risco. Também mostrou que a decisão ótima converge para a estratégia $1 / \mathrm{N}$ quando aumenta a incerteza em relação ao modelo probabilístico. [18] compara o desempenho de inúmeras estratégias de otimização de portfólio, tendo como benchmark a estratégia 1/N. Neste trabalho, analisaram o desempenho do modelo clássico de seleção de carteiras de Markowitz, também algumas das suas principais extensões e abordagens mais recentes baseadas nas crenças dos investidores sobre vários modelos de precificação de ativos. Além disso, os autores incluem abordagens que tentam minimizar a influência dos erros de estimação através da restrição do percentual investido em cada ativo ou concentrando-se inteiramente no portfólio de menor variância (mínimo de risco), ignorando totalmente os ganhos/perdas esperadas. Os resultados mostram que a carteira $1 / \mathrm{N}$ supera a maioria das outras estratégias envolvidas em termos de índice de Sharpe, equivalente certo e turnover $^{2}$, e o benchmark não foi consistentemente superado por nenhum dos modelos avaliados. Os autores explicam os resultados, afirmando que os erros na estimação dos parâmetros dos modelos de otimização superam os ganhos das metodologias mais avançadas.

Distributionally robust optimization (DRO) é a metodologia que estuda uma variante da restrição estocástica (1.3), onde a distribuição $\mathbb{P}^{0}$ é também sujeita a incerteza, e cujo objetivo é achar o vetor de decisão que garante a viabilidade da medida de valor do investidor para diferentes medidas de probabilidade $\mathbb{P}$. Mais precisamente:

$$
\mathbb{E}_{\mathbb{P}}[v(\mathbf{x}, \tilde{\mathbf{r}})] \geq \gamma \quad \forall \mathbb{P} \in \mathcal{P}
$$

\footnotetext{
${ }^{1}$ Também conhecido como estratégia de investimento uniforme ou ingênua, é quando o investidor divide sua riqueza uniformemente dentre todos os ativos disponíveis.

${ }^{2}$ No setor de investimentos, o turnover é definido como a porcentagem de uma carteira que é vendida em um determinado mês ou ano. Uma alto índice de turnover gera maiores custos de corretagem.
} 
ou equivalente

$$
\inf _{\mathbb{P} \in \mathcal{P}} \mathbb{E}_{\mathbb{P}}[v(\mathbf{x}, \tilde{\mathbf{r}})] \geq \gamma
$$

Neste tipo de problema, o pior caso é tomado sobre o conjunto de ambiguidade $\mathcal{P}$, isto é, a família de distribuições que são caracterizadas através de propriedades conhecidas em relação a distribuição $\mathbb{P}$. DRO é estudada desde os anos 50 com o trabalho [42], mas apenas após o surgimento de técnicas modernos de otimização robusta que esta metodologia passou a receber maior atenção [3], [9].

A principal característica da distributionally robust optimization está no conjunto de ambiguidade $\mathcal{P}$, pois este deve ser grande o suficiente para incluir a real distribuição dos parâmetros aleatórios e, ao mesmo tempo, suficientemente pequeno para excluir distribuições indesejadas que possam gerar soluções extremamente conservadoras, enquanto preserva a tratabilidade computacional, permitindo que o problema seja solucionado com algum solver comercial. Por exemplo [16] considera problemas DRO sob ambiguidade tanto na média quanto na covariância, e mostram como este tipo de problema pode ser resolvido em tempo polinomial quando o suporte das incertezas é convexo e compacto. [24] fornece uma formulação tratável quando DRO é aplicado em uma classe de problemas de otimização estocástica de dois estágios. Mais recentemente [53] fornece uma estrutura unificada para problemas DRO, onde o conjunto de ambiguidade é construído através de restrições probabilísticas e de momentos. Respeitando as condições de Slater, como também limitações em relação ao conjunto suporte, os autores provêm uma reformulação tratável do problema.

Este trabalho foca na construção do conjunto de ambiguidade $\mathcal{P}$, baseando-se somente nos dados observados, sem a necessidade de estimação ou especificação de qualquer parâmetro, tomando como base as condições de regularidade do trabalho [53].

As contribuições deste trabalho são:

1. Propomos a construção de conjuntos de ambiguidade poliédricos baseado somente em uma amostra de retornos. Nestes conjuntos, as relações entre variáveis são determinadas pelos dados de maneira não paramétrica, sendo assim livre de possíveis erros de especificação de um modelo estocástico.

2. Apresentamos um algoritmo para construção do conjunto e, dado o conjunto, uma reformulação computacionalmente tratável do problema de otimização de portfólio. 


\section{1 \\ Revisão Bibliográfica}

Distributionally robust optimization é uma poderosa metodologia de modelagem, pois diferentemente da otimização robusta clássica e programação estocástica, a contraparte distributionally robust (1.4) consegue explicitamente capturar a aversão ao risco e ambiguidade. Sendo esta última, uma questão extremamente relevante na teoria da decisão, pois existe o consenso que o tomador decisão possui baixa tolerância à incerteza em relação à distribuição $\mathbb{P}^{0}$, veja [23] e [22]. Logo, para estes agentes é racional decidir tendo em vista a pior distribuição possível, dado as informações disponíveis.

Recentemente foi mostrado que, sob hipóteses específicas em relação ao conjunto de ambiguidade $\mathcal{P}$ e a função objetivo $v$, a contraparte distributionally robust (1.4) herda a capacidade computacional da contraparte robusta clássica (1.1).

A história da distributionally robust optimization começou nos anos 50, onde muitos pesquisadores dependiam da construção de hipóteses com a finalidade de defender a elaboração de distribuições para o pior caso, que seriam modeladas em classes de problemas bem estruturados. Por exemplo, [42] estudou o problema do jornaleiro onde apenas a média e a variância da demanda é conhecida, enquanto [54] deriva da reformulação tratável para programação linear estocástica, onde somente o suporte e a média dos parâmetros aleatórios estavam disponíveis. Restrições de esperança distributionally robust podem eventualmente ser reduzidas em restrições esperança comuns, envolvendo distribuição de mistura, que é representável como uma combinação convexa de somente alguns membros do conjunto de ambiguidade. Caso possamos determinar explicitamente a distribuição da mistura, a restrição esperança subjacente se torna suscetível à técnicas de amostragem de Monte Carlo, por exemplo [31], [43] e [44].

Os trabalhos [25], [45] e [11] mostram que aproximações distributionally robust dependem do resultado de dualidade para problemas de momentos. [21] estudou problemas de distributionally robust optimization de quantis, onde mais tarde, seus métodos foram estendidos para restrições probabilísticas lineares e cônicas, onde apenas a média, matriz covariância e o suporte da distribuição de probabilidade subjacente são especificados, veja [12], [13], [14] e [55].

Os trabalhos [15] e [16] estudam reformulações robustas da forma (1.5), sob a hipótese que o conjunto de ambiguidade especifica o suporte e o conjunto de incerteza cônico para a média e a matriz covariância dos parâmetros aleatórios. O autor também fornece uma uma metodologia para construção 
de conjuntos de ambiguidade através dos dados históricos, utilizando a desigualdade de McDiarmid. [53] propõe uma estrutura conjuntos de ambiguidade padronizados e condições de regularidade, sobre os quais problemas de distributionally robust optimization são computacionalmente tratáveis. Programas distributionally robust lineares de dois estágios com informação de momentos de primeira e segunda ordem são investigados por [24]. Aproximações tratáveis para programas genéricos lineares distributionally robust de dois-estágios ou multi-estágios, dos quais assumem termos conhecimento apenas do suporte, média, matriz covariância e/ou desvios direcionais do problema de incerteza dos parâmetros derivam dos trabalhos [24] e [30].

O conceito de distributionally robust em relação a violação das restrições por famílias de conjuntos de ambiguidade paramétricos foi estendido por [2]. O artigo [50] mostra como calcular a persistência das variáveis de decisão binárias, isto é, a probabilidade destas variáveis assumirem o valor 1 em soluções ótimas. Existe uma forte conexão entre otimização robusta clássica, distributionally robust optimization e teoria sobre medida coerente de risco, veja [8], [37] e [52].

Apesar dos grandes avanços em distributionally robust optimization, ainda não foi desenvolvido uma estrutura unificada que permite modelar este tipo de problemas, sendo similar ao que ocorria com otimização robusta clássica, que em período anterior a publicação dos trabalhos [4] e [10] também não possuía uma metodologia para reformular classes genéricas de contraparte robustas.

\subsection{1 \\ Notação}

Para representar conjuntos, utilizamos letras maiúsculas em negrito A tal que $\mathbf{A}=\partial \mathbf{A} \cup \stackrel{\mathbf{o}}{\mathbf{A}}$, onde $\partial \mathbf{A}$ é a fronteira do conjunto, $\stackrel{\circ}{\mathbf{A}}$ seu interior e $\partial \mathbf{A} \cap \stackrel{\mathbf{A}}{\mathbf{A}}=\emptyset$. Letras minúscula, tais como $r$ representam elementos da reta, enquanto letras minúsculas em negrito $\mathbf{r}$ representam matrizes, $\mathbf{r}_{i}$ são vetores coluna e $\mathbf{r}^{\prime}$ e $\mathbf{r}_{\grave{1}}^{\prime}$ são as respectivas transpostas. Definimos $\tilde{\mathbf{r}}_{i}$ como vetores aleatórios e $\Theta_{N}=\left\{\hat{\mathbf{r}}_{1}, \hat{\mathbf{r}}_{2}, \hat{\mathbf{r}}_{3}, \ldots, \hat{\mathbf{r}}_{N}\right\}$ é a amostra com $N$ retornos observados, que foram extraídos de uma distribuição $\mathbb{P}$ desconhecida. Os conjuntos $\mathcal{M}_{+}\left(\mathbb{R}^{d}\right)$ e $\mathcal{P}_{0}\left(\mathbb{R}^{d}\right)$ representam os espaços de medidas não negativas e distribuição de probabilidade em $\mathbb{R}^{d}$, respectivamente. Se $\mathbb{P} \in \mathcal{P}_{0}\left(\mathbb{R}^{d} \times \mathbb{R}^{k}\right)$ é a distribuição de probabilidade conjunta de dois vetores aleatórios $\tilde{\mathbf{r}} \in \mathbb{R}^{d}$ $\tilde{\mathbf{u}} \in \mathbb{R}^{k}$, então $\prod_{\tilde{\mathbf{r}}} \mathbb{P} \in \mathcal{P}_{0}\left(\mathbb{R}^{d}\right)$ denota a distribuição marginal de $\tilde{\mathbf{r}}$ sobre $\mathbb{P}$. Estendemos a definição de conjuntos de ambiguidade $\mathcal{P} \subseteq \mathcal{P}_{0}\left(\mathbb{R}^{d} \times \mathbb{R}^{k}\right)$, estabelecendo $\prod_{\tilde{\mathbf{r}}} \mathcal{P}=\cup_{\mathbb{P} \in \mathcal{P}}\left\{\prod_{\tilde{\mathbf{r}}} \mathbb{P}\right\}$ para o cone próprio $\mathcal{K}$, isto é, convexo, 
fechado e pointed ${ }^{3}$, a relação $x \preceq \mathcal{K} y$ indica que $x-y \in \mathcal{K}$. Denotamos por $\mathbb{S}^{d}\left(\mathbb{S}_{+}^{d}\right)$ os cones de matrizes simétricas (positivas definidas) em $\mathbb{R}^{d} \times \mathbb{R}^{d}$. Para $\mathcal{K}, \mathcal{J} \in \mathbb{S}^{p}$, utilizamos $\mathcal{K} \preceq \mathcal{J}$ para abreviar $\mathcal{K} \preceq_{\mathbb{S}^{p}} \mathcal{J}$.

A função indicadora de um subconjunto $\mathbf{A}$ de um conjunto $\mathbf{X}$ é a função $\mathbb{I}_{\{\mathbf{x} \in \mathbf{A}\}}: \mathbf{X} \rightarrow\{0,1\}$, definida como

$$
\mathbb{I}_{\{\mathbf{x} \in \mathbf{A}\}}:=\left\{\begin{array}{lll}
1 & \text { se } & \mathbf{x} \in \mathbf{A} \\
0 & \text { se } & \mathbf{x} \notin \mathbf{A}
\end{array}\right.
$$

Sejam $\mathbf{r}, \mathbf{x} \in \mathbb{R}^{d}, b \in \mathbb{R}^{+}$e $\mathbf{A} \subset \mathbb{R}^{d}$.

A função $d(\mathbf{r}, \mathbf{x})=\sqrt{\sum_{i=1}^{d}\left(r_{i}-x_{i}\right)^{2}}$ é a métrica euclidiana. Quando dizemos que $d(\mathbf{r}, \mathbf{A})=b$, é o mesmo que $d(\mathbf{r}, \mathbf{x})=\sqrt{\sum_{i=1}^{d}\left(r_{i}-x_{i}\right)^{2}}=b \quad \forall \mathbf{x} \in$ A. 


\section{2}

\section{Base Teórica.}

Distributionally robust optimization é um modelo para tomada de decisão sob incerteza, onde o problema de incerteza dos dados é governado por uma distribuição de probabilidade, a qual também está sujeita a incerteza. Para lidar com este tipo de problema, assume-se que esta distribuição, que não conhecemos, pertence à um conjunto de ambiguidade que possui todas as distribuições possíveis, e que sejam compatíveis com as informações à priori do tomador de decisão. Contudo, há de ter cuidado na escolha destes conjuntos, pois há a necessidade de mantermos a tratabilidade computacional. $O$ trabalho [53] foca na representação de conjuntos de ambiguidade que são representados com restrições probabilísticas e de momentos. Apesar de conceitualmente simples, os autores mostram que estes conjuntos possibilitam a modelagem de indicadores estatísticos que ainda não foram considerados na literatura de otimização robusta, como por exemplo, momentos de alta ordem, medianas marginais, bem como medidas de dispersão baseadas no desvio médio absoluto e na função perda de Huber, que são estudadas em estatísticas robustas.

O artigo [53] propõe uma metodologia para DRO, com o intuito de desenvolver uma forma canônica para este tipo de problemas, que sob certas condições de regularidades conseguem resolver restrições do tipo (1.5) em tempo polinomial. Em suma, as condições são respeitadas se a função restrição $v$ é convexa e afim por partes, tanto nas variáveis de decisão quando nos vetores aleatórios, como também o conjunto de ambiguidade respeita a condição de aninhamento ${ }^{1}$. Os autores assumem que o conjunto de ambiguidade $\mathcal{P}$ em (1.4) é representável na seguinte forma padrão:

$$
\mathcal{P}=\left\{\begin{array}{ll}
\mathbb{P} \in \mathcal{P}_{0}\left(\mathbb{R}^{d} \times \mathbb{R}^{k}\right): & \mathbb{P}\left[(\tilde{\mathbf{r}}, \tilde{\mathbf{u}}) \in \mathcal{C}_{i}\right] \in\left[\underline{p_{i}}, \overline{p_{i}}\right] \\
& \mathbb{E}_{\mathbb{P}}[\mathbf{A} \tilde{\mathbf{r}}+\mathbf{B} \tilde{\mathbf{u}}]=\mathbf{b}, \\
& \forall i \in \mathfrak{I}
\end{array}\right\}
$$

onde $\mathbb{P}$ representa a distribuição de probabilidade conjunta do vetor aleatório $\tilde{\mathbf{r}} \in \mathbb{R}^{d}$, que aparece na função restrição (1.5), e algum vetor auxiliar $\tilde{\mathbf{u}} \in \mathbb{R}^{k}$.

${ }^{1}$ Tradução de condição Nesting. 
Assumem que $\mathbf{A} \in \mathbb{R}^{j \times d}, \mathbf{B} \in \mathbb{R}^{j \times k}, \mathbf{b} \in \mathbb{R}^{k}$ e $\mathcal{I}=\{1,2,3, \ldots, I\}$, onde os conjuntos $\mathcal{C}_{i}$ são definidos como

$$
\mathcal{C}_{i}=\left\{(\mathbf{r}, \mathbf{u}) \in \mathbb{R}^{d} \times \mathbb{R}^{k}: \mathbf{C}_{i} \mathbf{r}+\mathbf{D}_{i} \mathbf{u} \preceq \mathcal{K}_{i} \mathbf{c}_{i}\right\}
$$

com $\mathbf{C}_{i} \in \mathbb{R}^{L_{i} \times d}, \mathbf{D} \in \mathbb{R}^{L_{i} \times k}, \mathbf{c}_{i} \in \mathbb{R}^{L_{i}}$ e $\mathcal{K}_{i}$ é o cone adequado. Os autores assumem que $j$ ou $k$ sejam iguais a zero, e neste caso ou a esperança do (2.1) é nula ou o vetor $\tilde{\mathbf{u}}$ é ausente. Também assumem que $\underline{p}_{i}, \bar{p}_{i} \in[0,1]$ e $\underline{p}_{i} \leq \bar{p}_{i}$ para todo $i \in \mathcal{I}$.

Requerem que o conjunto de ambiguidade $\mathcal{P}$ satisfaça as seguintes condições de regularidade:

(C1) O conjunto $\mathcal{C}_{I}$ é limitado e possui probabilidade um, isto é, $\underline{p}_{I}=\bar{p}_{I}=1$

(C2) Existe uma distribuição de probabilidade $\mathbb{P} \in \mathcal{P}$ tal que $\mathbb{P}[(\tilde{\mathbf{r}}, \tilde{\mathbf{u}}) \in$ $\left.\mathcal{C}_{i}\right] \in\left(\underline{p}_{i}, \bar{p}_{i}\right)$, onde $\underline{p}_{i}<\bar{p}_{i}$ para todo $i \in \mathfrak{I}$.

A condição $(\mathrm{C} 1)$ garante que o conjunto de maior índice $\mathcal{C}_{I}$ possui o suporte do vetor aleatório $(\tilde{\mathbf{r}}, \tilde{\mathbf{u}})$. A segunda condição, também conhecida como condição de Slater, estipula que existe uma medida de probabilidade $\mathbb{P} \in \mathcal{P}$ que satisfaz a desigualdade estrita $\underline{p}_{i}<\bar{p}_{i}$ em (2.1), sempre que o intervalo for não degenerado.

Em relação a função restrição (1.5), requer que ela satisfaça a seguinte condição:

(C3) A função restrição $v(\mathbf{x}, \mathbf{r})$ pode ser escrita da seguinte forma:

$$
v(\mathbf{x}, \mathbf{r})=\max _{l \in \mathcal{L}} v_{l}(\mathbf{x}, \mathbf{r})
$$

onde $\mathcal{L}=\{1, \ldots, L\}$ e as funções $v_{l}: \mathbb{R}^{N} \times \mathbb{R}^{d} \rightarrow \mathbb{R}$ são da forma

$$
v_{l}(\mathbf{x}, \mathbf{r})=\mathbf{s}_{l}(\mathbf{r})^{T} \mathbf{x}+t_{l}(\mathbf{r})
$$

com $\mathbf{s}_{l}(\mathbf{r})=\mathbf{S}_{l} \mathbf{r}+\mathbf{s}_{l}, \mathbf{S}_{l} \in \mathbb{R}^{N \times d}$ e $\mathbf{s}_{l} \in \mathbb{R}^{N}, t_{l}(\mathbf{r})=\mathbf{t}_{l}^{T} \mathbf{r}+t_{l}, \mathbf{t}_{l} \in \mathbb{R}$. Onde $v$ deve ser convexa e linear por partes tanto nas variáveis de decisão quanto nos vetores aleatórios. A condição (C3) permitirá a utilização de técnicas de otimização robusta na reformulação da restrição semi-infinita que surge da reformulação dual da restrição (1.5). Contudo (C3) pode ser relaxada em inúmeras situações, veja [53] para mais detalhes.

Em relação aos conjuntos $\mathcal{C}_{i}, \forall i \in \mathfrak{I}$, assumimos que eles respeitam a seguinte condição:

(N) Para todo $i, i^{\prime} \in \mathfrak{I}, i \neq i^{\prime}$, temos $\mathcal{C}_{i} \Subset \mathcal{C}_{i^{\prime}}, \mathcal{C}_{i^{\prime}} \Subset \mathcal{C}_{i}$ ou $\mathcal{C}_{i} \cap \mathcal{C}_{i^{\prime}}=\emptyset$ A condição de aninhamento $(N)$, que é ilustrada ${ }^{2}$ na figura 2.1 , além de suficiente, é necessária para tratabilidade da restrição (1.5), e sua violação torna os problemas

${ }^{2}$ Figura retirada do artigo [53]. 
de otimização $\mathcal{N} \mathcal{P}$-hard, mesmo se o problema sem a restrição seja tratável. Contudo, os autores apresentam uma alternativa para tratar conjuntos de ambiguidade que violam a condição de aninhamento.
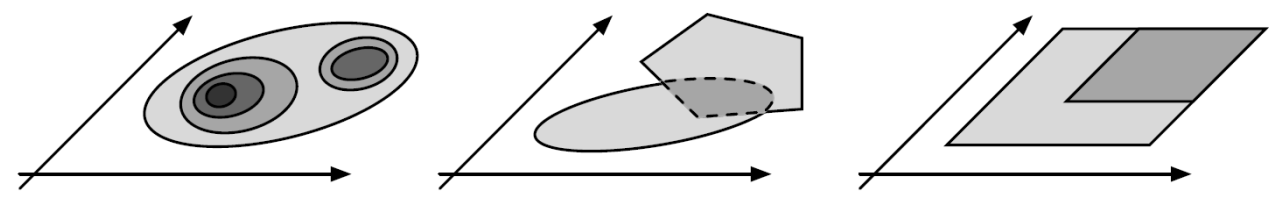

Figura 2.1: Ilustração da condição de aninhamento (N). Dentre os gráficos que mostram diferentes arranjos dos conjuntos $\mathcal{C}_{i}$, somente o primeiro à esquerda respeita a condição $(\mathrm{N})$, enquanto os demais violam. Fonte:[53]

Eles também apresentam inúmeros casos especiais do conjunto de ambiguidade (2.1), que através do teorema lifting, apresentado pelos autores, é possível codificar as informações sobre certos momentos de alta ordem do vetor aleatório $\tilde{\mathbf{r}}$ como por exemplo:

- Exemplo 1: Média

Assuma que $\mathrm{GE}_{\mathbb{P}_{0}}[\tilde{\mathbf{r}}] \preceq_{\mathcal{K}} \mathbf{f}$, para um cone próprio $\mathcal{K}$ e $\mathbf{G} \in \mathbb{R}^{M \times d}, \mathbf{f} \in \mathbb{R}^{M}$, e considere o seguinte exemplo para o conjunto de ambiguidade (2.1), o qual abrange um vetor aleatório auxiliar $\tilde{\mathbf{u}} \in \mathbb{R}^{M}$ :

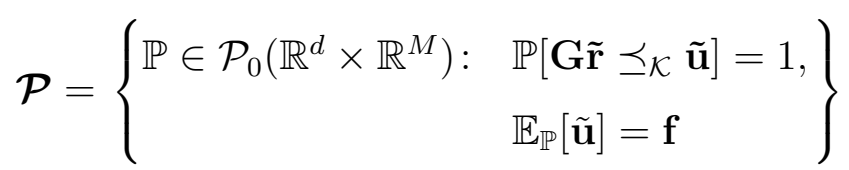

onde temos $\mathbb{P}_{0} \in \prod_{\tilde{\mathbf{r}}} \mathcal{P}=\left\{\mathbb{P} \in \mathcal{P}_{0}\left(\mathbb{R}^{d}\right): \mathbf{G} \mathbb{E}_{\mathbb{P}}[\tilde{\mathbf{r}}] \preceq_{\mathcal{K}} \mathbf{f}\right\}$

- Exemplo 2: Variância

Estabeleça $\boldsymbol{\mu}=\mathbb{E}_{\mathbb{P}_{0}}[\tilde{\mathbf{r}}]$ e assuma $\mathbb{E}_{\mathbb{P}_{0}}\left[(\tilde{\mathbf{r}}-\boldsymbol{\mu})(\tilde{\mathbf{r}}-\boldsymbol{\mu})^{T}\right] \preceq \boldsymbol{\Sigma}$ para $\boldsymbol{\Sigma} \in \mathbb{S}_{+}^{d}$. Considere o seguinte exemplo para o conjunto (2.1), o qual abrange uma matriz auxiliar $\tilde{\mathbf{U}} \in \mathbb{R}^{d \times d}$ :

$$
\mathcal{P}=\left\{\begin{array}{ll}
\mathbb{P} \in \mathcal{P}_{0}\left(\mathbb{R}^{d} \times \mathbb{R}^{d \times d}\right): & \mathbb{E}_{\mathbb{P}}[\tilde{\mathbf{r}}]=\boldsymbol{\mu}, \mathbb{E}_{\mathbb{P}}[\tilde{\mathbf{U}}]=\boldsymbol{\Sigma} \\
& \mathbb{P}\left(\left[\begin{array}{cc}
1 & (\tilde{\mathbf{r}}-\boldsymbol{\mu})^{T} \\
(\tilde{\mathbf{r}}-\boldsymbol{\mu}) & \tilde{\mathbf{U}}
\end{array}\right] \succeq \mathbf{0}\right)=1
\end{array}\right\}
$$

onde temos $\mathbb{P}_{0} \in \prod_{\tilde{\mathbf{r}}} \mathcal{P}=\left\{\mathbb{P} \quad \in \quad \mathcal{P}_{0}\left(\mathbb{R}^{d}\right): \mathbb{E}_{\mathbb{P}}[\tilde{\mathbf{r}}]=\right.$ $\left.\boldsymbol{\mu}, \mathbb{E}_{\mathbb{P}_{0}}\left[(\tilde{\mathbf{r}}-\boldsymbol{\mu})(\tilde{\mathbf{r}}-\boldsymbol{\mu})^{T}\right] \preceq \boldsymbol{\Sigma}\right\}$

- Exemplo 3: Coeficiente de Variação 
Assuma que $\sqrt{\mathbb{E}_{\mathbb{P}_{0}}\left[\left(\mathbf{f}^{T} \tilde{\mathbf{z}}-\mathbf{f}^{T} \boldsymbol{\mu}\right)^{2}\right]} / \mathbf{f}^{T} \boldsymbol{\mu} \leq \mathcal{V}$ para $\mathbf{f} \in \mathbb{R}^{d}, \mathcal{V} \in \mathbb{R}_{+}$e $\boldsymbol{\mu}=\mathbb{E}_{\mathbb{P 0} 0}[\tilde{\mathbf{r}}]$, tal que $\mathbf{f}^{T} \boldsymbol{\mu}>0$. Considere o seguinte exemplo para o conjunto de ambiguidade (2.1), o qual abrange um vetor aleatório auxiliar $\tilde{u} \in \mathbb{R}$ :

$$
\mathcal{P}=\left\{\begin{array}{ll}
\mathbb{P} \in \mathcal{P}_{0}\left(\mathbb{R}^{d} \times \mathbb{R}\right): & \mathbb{P}\left[\tilde{u} \geq\left(\mathbf{f}^{T} \tilde{\mathbf{r}}-\mathbf{f}^{T} \boldsymbol{\mu}\right)^{2}\right]=1, \\
& \mathbb{E}_{\mathbb{P}}[\tilde{\mathbf{r}}]=\boldsymbol{\mu}, \\
& \mathbb{E}_{\mathbb{P}}[\tilde{u}]=\mathcal{V}\left(\mathbf{f}^{T} \boldsymbol{\mu}\right)^{2}
\end{array}\right\}
$$

onde temos

$\mathbb{P}_{0} \in \prod_{\tilde{\mathbf{r}}} \mathcal{P}=\left\{\mathbb{P} \in \mathcal{P}_{0}\left(\mathbb{R}^{d}\right): \mathbb{E}_{\mathbb{P}}[\tilde{\mathbf{r}}]=\boldsymbol{\mu}, \sqrt{\mathbb{E}_{\mathbb{P}_{0}}\left[\left(\mathbf{f}^{T} \tilde{\mathbf{z}}-\mathbf{f}^{T} \boldsymbol{\mu}\right)^{2}\right]} / \mathbf{f}^{T} \boldsymbol{\mu} \leq \mathcal{V}\right\}$

- Informações de Momentos de Alta Ordem

Assuma que $\mathbb{E}_{\mathbb{P}_{0}}\left[f^{m / n}(\tilde{\mathbf{r}})\right] \leq \sigma$ para funções não negativas $f: \mathbb{R}^{d} \rightarrow \mathbb{R}_{+}$com epígrafe de representação cônica, enquanto $m, n \in \mathbb{N}$ com $m>n$. Considere o seguinte exemplo para o conjunto de ambiguidade (2.1), o qual abrange um vetor aleatório auxiliar $\tilde{\mathbf{u}}_{i, j} \in \mathbb{R}_{+}, i=1, \ldots, l, j=1, \ldots, 2^{l-i}$ e $\tilde{v} \in \mathbb{R}_{+}$.

$\mathcal{P}=\left\{\begin{array}{cc}\mathbb{P} \in \mathcal{P}_{0}\left(\mathbb{R}^{d} \times \mathbb{R}_{+}^{2^{l}}\right): & \mathbb{E}_{\mathbb{P}}[\tilde{v}]=\sigma, \\ & \mathbb{P}\left[\begin{array}{cc}\tilde{\mathbf{u}}_{i, j} \leq \sqrt{\tilde{\mathbf{u}}_{i-1,2 j-1} \tilde{\mathbf{u}}_{i-1,2 j}} & \forall i=1, \ldots, l, \\ f(\tilde{\mathbf{r}}) \leq \tilde{\mathbf{u}}_{l, 1} & \end{array}\right]=1, \ldots, 2^{l-i}\end{array}\right\}$

onde $l=\log _{2} m$ e $\tilde{\mathbf{u}}_{0, l}=\tilde{\mathbf{u}}_{l, 1}$ para $j=1, \ldots, 2^{l}-m ;=\tilde{v}$ para $j=$ $2^{l}-m+1, \ldots, 2^{l}-m+n ;=1$ caso contrário. Aplicando o teorema lifting, conclui-se que o conjunto que ambiguidade satisfaz

$\mathbb{P}_{0} \in \prod_{\tilde{\mathbf{r}}} \mathcal{P}=\left\{\mathbb{P} \in \mathcal{P}_{0}\left(\mathbb{R}^{d}\right): \mathbb{E}_{\mathbb{P}}\left[\mathbf{f}^{\mathbf{m} / \mathbf{n}}(\tilde{\mathbf{r}})\right] \leq \sigma\right\}$

Para maior detalhamento em relação aos conjuntos de ambiguidade apresentados, como também obtenção de mais exemplos, consulte do trabalho [53].

Em suma, esta metodologia requer a estimação de informações em relação aos momentos da variável aleatória, o que pode ser um problema, caso não tenhamos ao dispor informações suficientes para tal feito. Por isto, o propósito deste trabalho é apresentar uma metodologia completamente data driven, a qual será apresentada nos capítulos subsequentes, para tomada de decisão sob incerteza e ambiguidade. 


\section{Data-driven Portfolio Optimization Model - DPOM}

\section{1}

\section{Apresentação do Modelo}

Considere um problema de alocação de portfólio sob ambiguidade. Seja $(\Omega, \mathcal{F})$ um espaço mensurável e $\tilde{\mathbf{r}}: \Omega \rightarrow \mathbb{R}^{d}$ uma função $\mathcal{F}-$ mensurável que representa o retorno aleatório dos $d$ ativos.

$$
\max _{\mathbf{x} \in \mathcal{X}}\{\underbrace{\min _{\mathbb{P} \in \mathcal{P}} \mathbb{E}_{\mathbb{P}}[v(\mathbf{x}, \tilde{\mathbf{r}})]}_{(3.0)}\}
$$

onde,

$$
\mathcal{P}=\left\{\mathbb{P} \in \mathcal{M}_{+}\left(\mathbb{R}^{d}\right) \mid \mathbb{P}\left(\tilde{\mathbf{r}} \in \mathcal{C}_{i}\right) \in\left[\underline{p_{i}}, \overline{p_{i}}\right], \forall i \in \mathfrak{F}\right\},
$$

$\mathcal{C}_{i} \subsetneq \mathcal{R}, \forall i \in \mathfrak{F} \backslash\{0\}, \mathcal{R} \subset \mathcal{C}_{0}$ e $\mathfrak{F}=\{0,1, \cdots, I\}$, onde o conjunto $\chi$ representa as escolhas possíveis para o vetor de decisão. $v: \chi \times \mathcal{R} \rightarrow \mathbb{R}$ é a função payoff, côncava, que depende tanto do vetor $\mathbf{x} \in \mathbb{R}^{d}$ quanto das realizações de $\tilde{\mathbf{r}}$ dos parâmetros aleatórios. $\mathcal{R}$ é o suporte da variável aleatória $\tilde{\mathbf{r}}$, isto é, o menor conjunto $\mathcal{R} \subset \mathbb{R}^{d}$ tal que $\mathbb{P}(\tilde{\mathbf{r}} \in \mathcal{R})=1, \mathcal{P}$ é o conjunto de ambiguidade, onde $\mathbb{P}$ é a distribuição de probabilidade do vetor $\tilde{\mathbf{r}}$, e $\underline{p}_{i}, \bar{p}_{i} \in[0,1]$ e $\underline{p}_{i} \leq \bar{p}_{i}$ para todo $i \in \mathfrak{F} .\{\mathcal{C}\}_{i \in \mathfrak{F}}$ é uma família de conjuntos convexos, dos quais existe uma vasta e crescente literatura que sugere diferentes meios para construí-los. Cada uma destas formulações enfrentam trade-offs distintos, que geram vantagens e desvantagens em relação a cada tipo de problema, com diferentes níveis de robustez em relação a garantia de não violação das restrições, onde alguma formulação irregular destes conjuntos pode gerar soluções excessivamente conservadoras ou conduzir à intratabilidade do problema.

\section{2}

Metodologia para Construção de um Conjunto de Ambiguidade Poliédrico: Uma Abordagem Data-Driven

Assumimos as condições (C1), (C2) e a condição de aninhamento (N) do artigo [53] para garantir a tratabilidade do nosso problema, isto é, requeremos que 
o conjunto de ambiguidade $\mathcal{P}$ satisfaça as seguintes condições de regularidade, onde:

(C1) $\mathrm{O}$ conjunto $\mathcal{C}_{0}$ é limitado e possui probabilidade um, isto é, $\underline{p}_{0}=\bar{p}_{0}=1$

(C2) Existe uma distribuição de probabilidade $\mathbb{P} \in \mathcal{P}$ tal que $\mathbb{P}\left(\tilde{\mathbf{r}} \in \mathcal{C}_{i}\right) \in$ $\left(\underline{p}_{i}, \bar{p}_{i}\right)$, onde $\underline{p}_{i}<\bar{p}_{i}$ para todo $i \in \mathfrak{F} \backslash\{0\}$.

Em relação aos conjuntos $\mathcal{C}_{i}, \forall i \in \mathfrak{F}$, assumimos que eles respeitam a seguinte condição:

(N) Para todo $i, i^{\prime} \in \mathfrak{F}, i \neq i^{\prime}$, temos $\mathcal{C}_{i} \Subset \mathcal{C}_{i^{\prime}}, \mathcal{C}_{i^{\prime}} \Subset \mathcal{C}_{i}$ ou $\mathcal{C}_{i} \cap \mathcal{C}_{i^{\prime}}=\emptyset$

A primeira condição garante que o suporte do vetor aleatório $\tilde{\mathbf{r}}$ esteja contido no conjunto de incerteza de menor índice $\mathcal{C}_{0}$. A segunda condição estipula que existe uma distribuição de probabilidade $\mathbb{P} \in \mathcal{P}$ que satisfaz os limites estritos de probabilidade em (3.2), sempre que o intervalo $\left[p_{i}, \overline{p_{i}}\right]$ for não degenerado.

Este trabalho propõe modelar a incerteza dentro do problema, ao restringir que os parâmetros aleatórios pertençam ao conjunto de incerteza poliedral $\mathcal{C}_{i}$, $\forall i \in \mathfrak{F}$, descritos na definição 3.1. Como também, sugerimos a utilização da metodologia indicada na definição 3.4 para construção dos intervalos, descritos no conjunto de ambiguidade 3.2 .

Definição 3.1 Seja $\mathcal{C}_{i}$ o fecho convexo de $\mathbf{A}_{i}, \forall i \in \mathfrak{F}$, onde $\left\{\mathbf{A}_{i}\right\}_{i \in \mathfrak{F}}$ é uma família de subconjuntos de $\mathbb{R}^{d}$, e $\mathbf{A}_{0}$ é o conjunto de todos os retornos observados dos ativos, e os $\mathbf{A}_{j}, \forall j \in \mathfrak{F} \backslash\{0\}$, são definidos da seguinte forma:

$$
\begin{aligned}
\mathbf{A}_{0} & =\left\{\hat{\mathbf{r}}_{1}, \hat{\mathbf{r}}_{2}, \hat{\mathbf{r}}_{3}, \ldots, \hat{\mathbf{r}}_{N}\right\} \\
\mathbf{A}_{1} & =\mathbf{A}_{0} \backslash\left(\partial \mathcal{C}_{0} \cap \mathbf{A}_{0}\right) \\
\mathbf{A}_{2} & =\mathbf{A}_{1} \backslash\left(\partial \mathcal{C}_{1} \cap \mathbf{A}_{1}\right) \\
\vdots & \\
\mathbf{A}_{I} & =\mathbf{A}_{I-1} \backslash\left(\partial \mathcal{C}_{I-1} \cap \mathbf{A}_{I-1}\right) .
\end{aligned}
$$

Teorema 3.2 Seja $\left\{\mathcal{C}_{i}\right\}_{i \in \mathfrak{F}}$ uma família de fechos convexos da Definição 3.1, então:

$$
\mathcal{C}_{I} \Subset \mathcal{C}_{I-1} \Subset \mathcal{C}_{I-2} \Subset \ldots \Subset \mathcal{C}_{0}
$$

Demonstração. Primeiro mostraremos que $\left(\mathcal{C}_{I-1} \cap \mathbf{A}_{I-1}\right) \neq \emptyset$.

Pela definição de fecho convexo, $\mathbf{r} \in \mathbf{A}_{I-1} \Rightarrow \mathbf{r} \in \mathcal{C}_{I-1}$, então para qualquer $\mathbf{r} \in \mathbf{A}_{I-1}$ temos $\mathbf{r} \in \partial \mathcal{C}_{I-1}$ ou $\mathbf{r} \in \mathcal{C}_{I-1}^{o}$ e pelo fato do fecho convexo ser um conjunto compacto, temos $\left(\mathcal{C}_{I-1} \cap \mathbf{A}_{I-1}\right) \neq \emptyset \Rightarrow \mathbf{A}_{I} \subset \mathbf{A}_{I-1} \Rightarrow \mathcal{C}_{I} \subset \mathcal{C}_{I-1}$.

Para provar que $\mathcal{C}_{I} \Subset \mathcal{C}_{I-1}$ basta mostrar que $\left(\mathbf{A}_{I} \cap \partial \mathcal{C}_{I-1}\right)=\emptyset$. 
Se $\exists \mathbf{r} \in \mathbf{A}_{I}$ tal que $d\left(\mathbf{r}, \partial \mathcal{C}_{I-1}\right)=0 \Rightarrow \mathbf{r} \in\left(\partial \mathcal{C}_{I-1} \cap \mathbf{A}_{I-1}\right)$, mas como $\mathbf{A}_{I}=$ $\mathbf{A}_{I-1} \backslash\left(\partial \mathcal{C}_{I-1} \cap \mathbf{A}_{I-1}\right)$ então $\mathbf{r} \notin \mathbf{A}_{I}$. Logo se $\mathcal{C}_{I-1}$ não está compactamente contido em $\mathcal{C}_{I}$ então $\left(\mathbf{A}_{I} \cap \partial \mathcal{C}_{I}\right) \neq \emptyset$, logo $\mathcal{C}_{I-1} \Subset \mathcal{C}_{I}$.

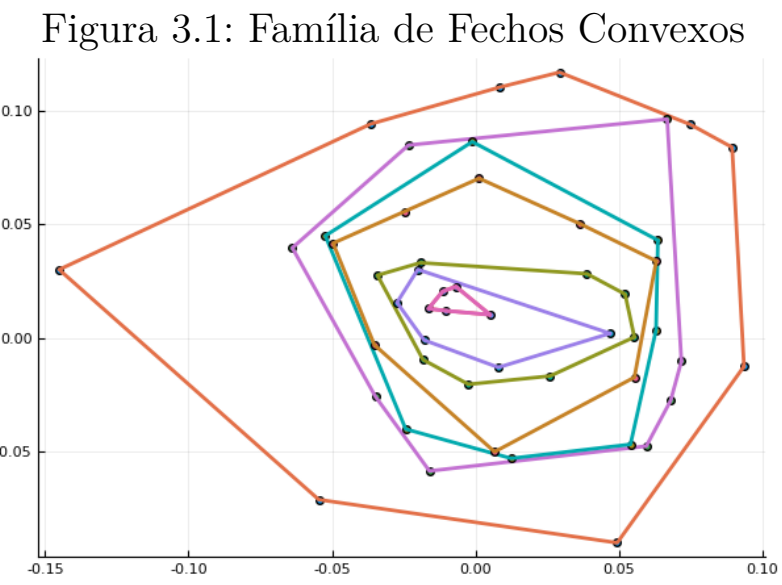

Família de conjuntos de incerteza construídos como na Definição 3.1.

Com isto, o teorema 3.2 garante $(\mathrm{N})$ seja satisfeita.

Definição 3.3 Para construção dos intervalos $\left[\underline{p}_{i}, \bar{p}_{i}\right]$ de (3.2), utilizamos a aproximação Normal, que é satisfeita para um $N$ suficientemente grande ${ }^{1}$. Consideramos $\hat{\mathbf{p}}$ a distribuição empírica, isto é

$$
\hat{p}_{i}=\frac{1}{N} \sum_{j=1}^{N} \mathbb{I}_{\left\{\hat{\mathbf{r}}_{j} \in \mathbf{A}_{i}\right\}} \quad \forall i \in \mathfrak{F}
$$

Pelo Teorema Central do Limite temos que, para um tamanho de amostra grande, podemos considerar que a proporção amostral $\hat{p}_{i}$ possui aproximadamente distribuição Normal com média $p_{i}$ e variância $\frac{p_{i}\left(1-p_{i}\right)}{N}$. Desse modo segue que

$$
\hat{p}_{i} \sim \mathcal{N}\left(p_{i}, \frac{p_{i}\left(1-p_{i}\right)}{N}\right) \quad \forall i \in \mathfrak{F}
$$

Como não conhecemos o parâmetro $\mathbf{p}$, e pelo fato de $N$ ser suficientemente grande, podemos substituí-lo por $\hat{\mathbf{p}}$, com isto temos,

$$
Z=\frac{\hat{p}_{i}-p_{i}}{\sqrt{\frac{p_{i}\left(1-p_{i}\right)}{N}}} \sim \mathcal{N}(0,1) \quad \forall i \in \mathfrak{F}
$$

\footnotetext{
${ }^{1}$ Assumimos que uma amostra de tamanho $N$ é suficientemente grande se: $N p \geq 5$
} 
Logo, considerando o mesmo procedimento, construímos o intervalo de confiança com $100(1-\alpha) \%$ nível de significância para $p_{i}$ :

$$
I C_{(1-\alpha)}=\left[\hat{p}_{i}-z_{(1-\alpha / 2)} \sqrt{\frac{\hat{p}_{i}\left(1-\hat{p}_{i}\right)}{N}}, \hat{p}_{i}+z_{(1-\alpha / 2)} \sqrt{\frac{\hat{p}_{i}\left(1-\hat{p}_{i}\right)}{N}}\right] \quad \forall i \in \mathfrak{F}
$$

onde

$$
\underline{p}_{i}=\hat{p}_{i}-z_{(1-\alpha / 2)} \sqrt{\frac{\hat{p}_{i}\left(1-\hat{p}_{i}\right)}{N}} \quad \text { e } \quad \bar{p}_{i}=\hat{p}_{i}+z_{(1-\alpha / 2)} \sqrt{\frac{\hat{p}_{i}\left(1-\hat{p}_{i}\right)}{N}} \quad \forall i \in \mathfrak{F}
$$

Em relação a condição (C2), a metodologia de construção do intervalo de confiança na definição 3.3 garante que ela seja satisfeita.

\section{3}

\section{Reformulação do problema.}

Teorema 3.4 O problema de alocação de portfólio (3.1) pode ser escrito da seguinte forma:

$$
\begin{aligned}
& \max _{\mathbf{x} \in \mathcal{X}, \boldsymbol{\lambda}, \kappa} \sum_{i \in \mathfrak{F}}\left(\underline{p}_{i} \lambda_{i}-\bar{p}_{i} \kappa_{i}\right) \\
& \text { s.a. } \\
& v(\mathbf{x}, \tilde{\mathbf{r}})-\sum_{i \in \mathfrak{F}} \mathbb{I}_{\left\{\mathbf{r} \in \mathcal{C}_{i}\right\}}\left(\lambda_{i}-\kappa_{i}\right) \geq 0 \\
& \lambda_{i}, \kappa_{i} \geq 0
\end{aligned}
$$

onde II é a função indicadora.

Demonstração. Reescrevemos o problema de minimização (3.0), que esta dentro de do problema (3.1) da seguinte forma:

$$
\begin{array}{ll}
\min _{\mathbb{P} \in \mathcal{M}_{+}} \int_{\mathcal{C}_{0}} v(\mathbf{x}, \tilde{\mathbf{r}}) d \mathbb{P} & \\
\text { s.a. } & \forall i \in \mathfrak{F} \\
\int_{\mathcal{C}_{0}} \mathbb{I}_{\left\{\mathbf{r} \in \mathcal{C}_{i}\right\}} d \mathbb{P} \geq \underline{p}_{i} & \forall i \in \mathfrak{F} \\
\int_{\mathcal{C}_{0}} \mathbb{I}_{\left\{\mathbf{r} \in \mathcal{C}_{i}\right\}} d \mathbb{P} \leq \bar{p}_{i} &
\end{array}
$$

O Lagrangiano do problema (3.5) problema é: 


$$
\begin{aligned}
L(\mathbb{P}, \boldsymbol{\kappa}, \boldsymbol{\lambda})= & \int_{\mathcal{C}_{0}} v(\mathbf{x}, \tilde{\mathbf{r}}) d \mathbb{P}-\int_{\mathcal{C}_{0}} \sum_{i \in \mathfrak{F}}\left[\lambda_{i}\left(\mathbb{I}_{\left\{\mathbf{r} \in \mathcal{C}_{i}\right\}}-\underline{p}_{i}\right)\right] d \mathbb{P} \\
& \quad+\int_{\mathcal{C}_{0}} \sum_{i \in \mathfrak{F}}\left[\kappa_{i}\left(\mathbb{I}_{\left\{\mathbf{r} \in \mathcal{C}_{i}\right\}}-\bar{p}_{i}\right)\right] d \mathbb{P} \\
= & \int_{\mathcal{C}_{0}} v(\mathbf{x}, \tilde{\mathbf{r}}) d \mathbb{P}-\int_{\mathcal{C}_{0}} \sum_{i \in \mathfrak{F}}\left[\mathbb{I}_{\left\{\mathbf{r} \in \mathcal{C}_{i}\right\}}\left(\lambda_{i}-\kappa_{i}\right)\right] d \mathbb{P} \\
& +\int_{\mathcal{C}_{0}} \sum_{i \in \mathfrak{F}}\left(\lambda_{i} \underline{p}_{i}-\kappa_{i} \bar{p}_{i}\right) d \mathbb{P} \\
= & \int_{\mathcal{C}_{0}}\left[v(\mathbf{x}, \tilde{\mathbf{r}})-\sum_{i \in \mathfrak{F}} \mathbb{I}_{\left\{\mathbf{r} \in \mathcal{C}_{i}\right\}}\left(\lambda_{\mathbf{i}}-\kappa_{\mathbf{i}}\right)\right] d \mathbb{P}+\sum_{i \in \mathfrak{F}}\left(\lambda_{i} \underline{p}_{i}-\kappa_{i} \bar{p}_{i}\right) .
\end{aligned}
$$

Logo,

$$
\begin{aligned}
\max _{\boldsymbol{\lambda}, \boldsymbol{\kappa}}\left\{\min _{\mathbb{P} \in \mathcal{M}_{+}} L(\mathbb{P}, \boldsymbol{\kappa}, \boldsymbol{\lambda})\right\}= & \max _{\boldsymbol{\lambda}, \boldsymbol{\kappa}}\left\{\min _{\mathbb{P} \in \mathcal{M}_{+}} \int_{\mathcal{C}_{0}}[v(\mathbf{x}, \tilde{\mathbf{r}})-\right. \\
& \left.\left.\sum_{i \in \mathfrak{F}} \mathbb{I}_{\left\{\mathbf{r} \in \mathcal{C}_{i}\right\}}\left(\lambda_{i}-\kappa_{i}\right)\right] d \mathbb{P}+\sum_{i \in \mathfrak{F}}\left(\lambda_{i} \underline{p}_{i}-\kappa_{i} \bar{p}_{i}\right)\right\} .
\end{aligned}
$$

Note que

$$
\begin{aligned}
& \min _{\mathbb{P} \in \mathcal{M}_{+}} \int_{\mathcal{C}_{0}} v(\mathbf{x}, \tilde{\mathbf{r}})-\sum_{i \in \mathfrak{F}} \mathbb{I}_{\left\{\mathbf{r} \in \mathcal{C}_{i}\right\}}\left(\lambda_{i}-\kappa_{i}\right) d \mathbb{P}= \\
& \qquad \begin{array}{cl}
0, & \text { se } \int_{\mathcal{C}_{0}} v(\mathbf{x}, \tilde{\mathbf{r}})-\sum_{i \in \mathfrak{F}} \mathbb{I}_{\left\{\mathbf{r} \in \mathcal{C}_{i}\right\}}\left(\lambda_{i}-\kappa_{i}\right) d \mathbb{P} \geq 0 \\
-\infty, & \text { caso contrário }
\end{array}
\end{aligned}
$$

Dado que (C1) é respeitado, temos que toda medida possível dentro deste problema é naturalmente identificada como uma medida de probabilidade $\mathbb{P} \in \mathcal{P}$, cujo suporte está em $\mathcal{C}_{0}$. Na maximização de $\min _{\mathbb{P} \in \mathcal{M}_{+}} L(\mathbb{P}, \boldsymbol{\kappa}, \boldsymbol{\lambda})$, precisamos apenas considerar os valores de $\boldsymbol{\lambda}, \boldsymbol{\kappa}$ dos quais (3.6) é diferente de $-\infty$. Então, segue pelo teorema da dualidade forte [45], que é aplicável devido à condição (C2), também conhecida como condição de qualificação de Slater [48], que o problema dual de (3.5) possui a mesma solução ótima que o problema primal (3.0). Logo, o dual do problema de momentos é 


$$
\begin{aligned}
& \max _{\lambda, \kappa} \sum_{i \in \mathfrak{F}}\left(\lambda_{i} \underline{p}_{i}-\kappa_{i} \bar{p}_{i}\right) \\
& \text { s.a. } \\
& v(\mathbf{x}, \tilde{\mathbf{r}})-\sum_{i \in \mathfrak{F}} \mathbb{I}_{\left\{\mathbf{r} \in \mathcal{C}_{i}\right\}}\left(\lambda_{i}-\kappa_{i}\right) \geq 0 \\
& \lambda_{i}, \kappa_{i} \geq 0
\end{aligned}
$$

Com isto, podemos reescrever o problema (3.1):

$$
\begin{aligned}
\max _{\mathbf{x} \in \mathcal{X}}\left\{\max _{\lambda, \kappa} \sum_{i \in \mathfrak{F}}\left(\lambda_{i} \underline{p}_{i}-\kappa_{i} \bar{p}_{i}\right)\right. \\
\quad \text { s.a. } \\
\left.\quad v(\mathbf{x}, \tilde{\mathbf{r}})-\sum_{i \in \mathfrak{F}} \mathbb{I}_{\left\{\mathbf{r} \in \mathcal{C}_{i}\right\}}\left(\lambda_{i}-\kappa_{i}\right) \geq 0, \quad \lambda_{i}, \kappa_{i} \geq 0\right\} \quad \forall \mathbf{r} \in \mathcal{C}_{0}
\end{aligned}
$$

que é equivalente à

$$
\begin{array}{ll}
\max _{\mathbf{x} \in \mathcal{\chi}, \lambda, \kappa} & \sum_{i \in \mathfrak{F}}\left(\lambda_{i} \underline{p}_{i}-\kappa_{i} \bar{p}_{i}\right) \\
\text { s.a. } & \\
v(\mathbf{x}, \mathbf{r})-\sum_{i \in \mathfrak{F}} \mathbb{I}_{\left\{\mathbf{r} \in \mathcal{C}_{i}\right\}}\left(\lambda_{i}-\kappa_{i}\right) \geq 0 & \forall \mathbf{r} \in \mathcal{C}_{0} \\
\lambda_{i}, \kappa_{i} \geq 0 &
\end{array}
$$

\section{4}

\section{Tratabilidade}

A ideia de uma contraparte robusta está relacionada ao tratamento computacional de um problema robusto, algo que ainda não é possível em relação ao problema (3.7) por causa da existência da função indicadora na da restrição (3.8).

Para facilitar nossa notação, assumimos as definições dos conjuntos de índices proposto pelo trabalho [53], isto é, denotamos $\mathscr{A}(i)=\{i\} \cup\left\{i^{\prime} \in \mathfrak{F}: \mathcal{C}_{i} \Subset \mathcal{C}_{i^{\prime}}\right\}$ (Ascendentes), e $\mathscr{D}(i)=\left\{i^{\prime} \in \mathfrak{F}: \mathcal{C}_{i^{\prime}} \Subset \mathcal{C}_{i}\right\}$ (Descendentes). 
Figura 3.2: Conjuntos ascendentes e descendentes do conjunto de incerteza $\mathcal{C}_{2}$
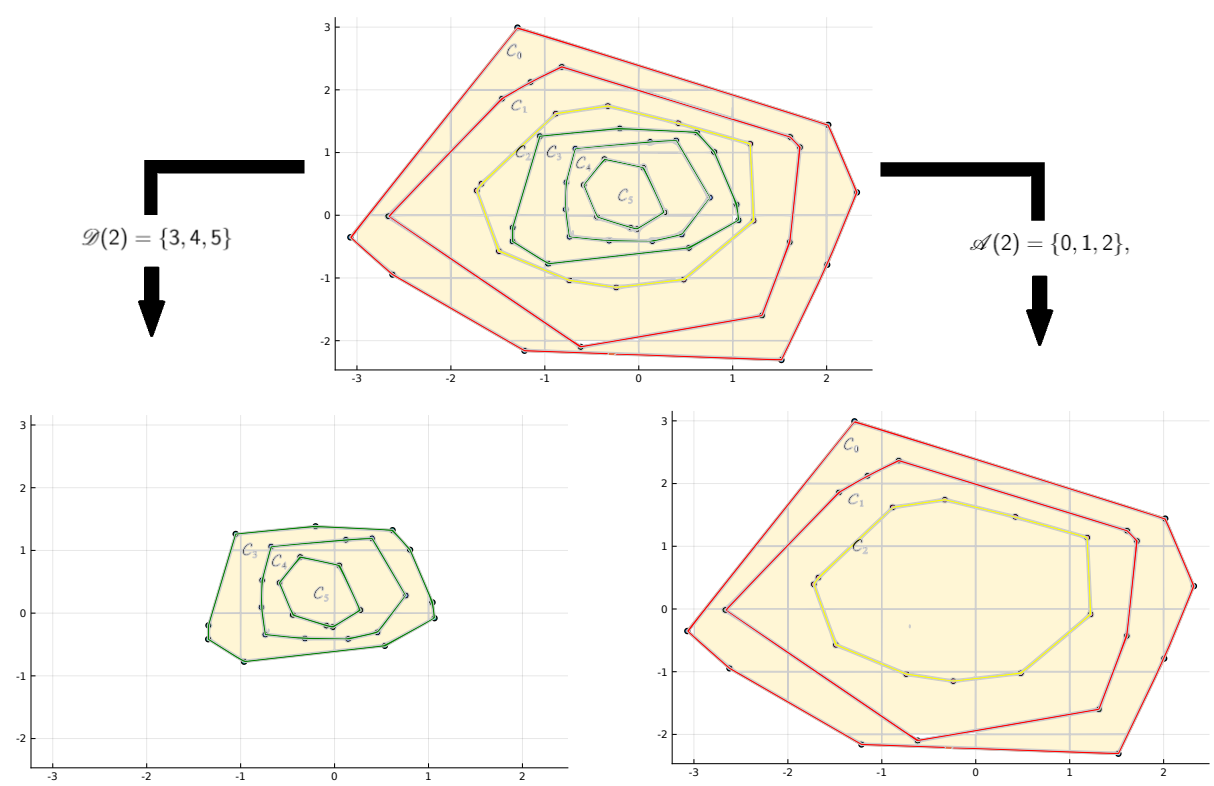

Na figura da esquerda mostra os conjuntos descendentes do conjunto de incerteza $\mathcal{C}_{2}$ enquanto o da direita os conjuntos ascendentes.

Devido a condição $(\mathrm{N})$, é possível particionar o suporte $\mathcal{C}_{0}$ em $I$ conjuntos disjuntos não vazios $\overline{\mathcal{C}}_{i}=\mathcal{C}_{i} \backslash \cup_{i^{\prime} \in \mathscr{D}(i)} \mathcal{C}_{i^{\prime}}$, onde $i \in \mathfrak{F}$ e $\mathscr{D}(i)$ denota o conjunto de índices de subconjuntos estritos de $\mathcal{C}_{i}$.

Figura 3.3: Conjuntos $\overline{\mathcal{C}_{i}}$ gerados com o particionamento do suporte $\mathcal{C}_{0}$

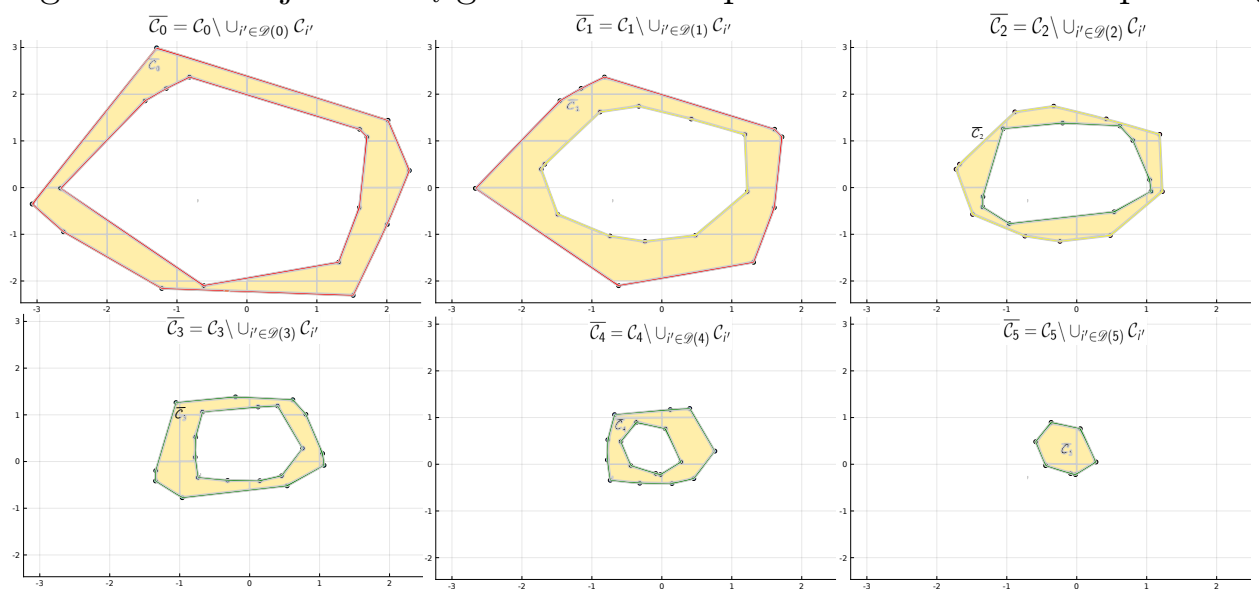

Família de conjuntos gerados com a metodologia apresentada de particionamento do suporte $\mathcal{C}_{2}$.

Logo, a restrição (3.8) do problema (3.7) é equivalente ao seguinte conjunto 
de restrições:

$$
v(\mathbf{x}, \mathbf{r})-\sum_{i^{\prime} \in \mathscr{A}(i)}\left(\lambda_{i^{\prime}}-\kappa_{i^{\prime}}\right) \geq 0 \quad \forall \mathbf{r} \in \overline{\mathcal{C}}_{i}, \forall i \in \mathfrak{F}
$$

como a desigualdade acima é válida para todo $\mathbf{r} \in \overline{\mathcal{C}}_{i}$, para todo $i \in \mathfrak{F}$, temos que (3.9) é equivalente a

$$
\min _{r \in \overline{\mathcal{C}}_{i}}\{v(\mathbf{x}, \mathbf{r})\} \geq \sum_{i^{\prime} \in \mathscr{A}(i)}\left(\lambda_{i^{\prime}}-\kappa_{i^{\prime}}\right) \quad \forall i \in \mathfrak{F}
$$

A expressão dentro da minimização herda a concavidade da função $v$, e por isto a solução ótima do problema é atingida nos vértices do conjunto poliedral $\overline{\mathcal{C}}_{i}$, que devido a condição $(\mathrm{N})$, coincide com os vértices do conjunto $\mathcal{C}_{i}$, que são equivalentes ao pontos extremos de $\mathbf{A}_{i}$. Com isto, temos

$$
\min _{\mathbf{r} \in \mathbf{A}_{i}}\{v(\mathbf{x}, \mathbf{r})\} \geq \sum_{i^{\prime} \in \mathscr{A}(i)}\left(\lambda_{i^{\prime}}-\kappa_{i^{\prime}}\right) \quad \forall i \in \mathfrak{F}
$$

Assim, o problema (3.1) é equivalente a:

$$
\begin{aligned}
& \max _{\mathbf{x} \in \mathcal{X}, \boldsymbol{\lambda}, \kappa} \sum_{i \in \mathfrak{F}}\left(\lambda_{i} \underline{p}_{i}-\kappa_{i} \bar{p}_{i}\right) \\
& \text { s.a } \\
& v(\mathbf{x}, \mathbf{r})-\sum_{i^{\prime} \in \mathscr{A}(i)}\left(\lambda_{i^{\prime}}-\kappa_{i^{\prime}}\right) \geq 0 \quad \forall \mathbf{r} \in \mathbf{A}_{i}, \forall i \in \mathfrak{F} \\
& \lambda_{i}, \kappa_{i} \geq 0
\end{aligned}
$$

Logo, o problema (3.10) é tratável com Solvers comerciais.

\section{5}

\section{Hipóteses}

Neste trabalho, desenvolvemos um problema de seleção de portfólio onde o tomador de decisão deve selecionar a estratégia antes de observar os valores assumidos pelos parâmetros aleatórios. Seja $\tilde{\mathbf{r}}_{t}$ o retorno dos ativos no período $t$, o qual é uma variável aleatória, $\mathbb{E}_{\mathbb{P}}\left[v\left(\mathbf{x}, \tilde{\mathbf{r}}_{t}\right) \mid \Theta_{N}\right]$ é o retorno esperado do portfólio com base nas informações obtidas na amostra de tamanho $s$, representada por $\Theta_{N}$. Consideramos que o modelo realiza alocações diariamente, e para cada $t$, a figura 3.4 mostra a estrutura de estimação das variáveis e a implementação do modelo. 
Figura 3.4: Diagrama de estimação e período implementação do modelo

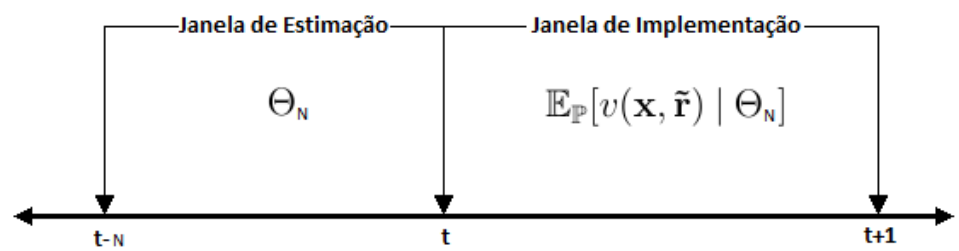

Consideramos que as decisões de alocação são feitas no início do período $t$, tendo como base as informações obtidas através da amostra de realizações observadas $\Theta_{N}=\left\{\hat{\mathbf{r}}_{t-1}, \hat{\mathbf{r}}_{t-2}, \ldots, \hat{\mathbf{r}}_{t-N}\right\}$, no início do período $t+1$, a alocação é desfeita.

Chamamos de Chull $_{N}$, cada estratégia do DPOM quando utilizamos uma amostra de tamanho $N$ para implementação do nosso modelo. 


\section{4}

\section{Aplicação do Modelo}

\section{1}

\section{Estudo de Caso}

Nesta seção, demostraremos como a nossa metodologia de construção de conjuntos de incerteza pode ser utilizada em um problema de otimização de portfólio. Esta classe de problemas são extremamente desafiadores, pois estamos interessados em maximizar o retorno do portfólio em um período, com base apenas nas informações obtidas através da amostra das realizações observadas do retornos dos ativos que irão compor este portfólio. Seja o problema de maximização de portfólio:

$$
\begin{aligned}
& \max _{\mathbf{x}_{t}}\left\{\min _{\mathbb{P} \in \mathcal{P}} \mathbb{E}_{\mathbb{P}}\left[\tilde{\mathbf{r}}_{t}^{\prime} \mathbf{x}_{t}\right]\right\} \\
& \text { s.a. } \\
& \mathbf{x}_{t} \in \chi
\end{aligned}
$$

Assumimos que a função $v\left(\mathbf{x}_{t}, \tilde{\mathbf{r}}_{t}\right)=\tilde{\mathbf{r}}_{t}^{\prime} \mathbf{x}_{t}$, o conjunto das escolhas possíveis para o vetor de decisão é definido por $\chi=\left\{\mathbf{x}_{t} \in \mathbb{R}_{+}^{d} \mid \sum_{i=1}^{d} x_{i, t}=1\right\}$, o vetor dos retornos dos ativos é representado por $\tilde{\mathbf{r}}_{t}^{\prime}=\left[\tilde{r}_{t, 1}, \tilde{r}_{t, 2}, \ldots, \tilde{r}_{t, d}\right]$ e $\mathbf{x}_{t}^{\prime}=$ $\left[x_{t, 1}, x_{t, 2}, \ldots, x_{t, d}\right]$ é o vetor de variáveis de decisão, onde cada $x_{t, i}$ corresponde ao parcela da riqueza que será alocada no ativo $i$ no início do dia $t$, e $d$ é o número total de ativos disponíveis.

Assumimos que o conjunto poliedral que descreve a incerteza é construído, usando a definição 3.1, tendo como base as informações disponíveis na amostra $\Theta_{N}$ antes que a otimização robusta seja implementada.

\section{2}

\section{Experimento Numérico}

Avaliamos a estrutura do nosso modelo de otimização usando dados diários do Industry Portfólios com 2 ativos, em um horizonte de 20 anos (20/03/1998 até 29/03/2018), totalizando 5040 observações, extraídas da base de dados do 
Kenneth French[29]. A carteira com 2 ativos foi extraída aleatoriamente da carteira com 5 ativos e os dados da série histórica do índice SP500 e Down Jones foram extraído do site Yahoo! Finance[46].

Seja o dia inicial $t_{0}$, consideramos que as decisões de alocação são realizadas diariamente, e comparamos o desempenho do algorítimo, assumindo diferentes amostras de retornos observados $\Theta_{N}$ onde $N=$ $\{5,10,15,30,50,100,252,350,400,450,500,600\}$, que foram escolhidos arbitrariamente.

Implementamos o algorítimo de otimização de portfólio sem considerar os custos de transação. Foi utilizado o solver Xpress[51] para resolver os problemas de otimização, como também a linguagem de programação Julia[28] e JuMP ${ }^{1}$ para formular o algorítimo utilizado na solução computacional.

Como benchmark ${ }^{2}$, utilizamos as estratégias de gestão passiva, onde o portfólio é completamente investido no índice SP500, como também a estratégia naive $^{3} 1 / \mathrm{d}$, que investe a mesma fração do portfólio em cada um dos $d$ ativos disponíveis em cada data de rebalanceamento. Existe uma vasta literatura que apresenta a superioridade da estratégia naive, pois esta consistentemente gera performances superiores à grande parte dos modelos de alocação de portfólio, ver [7] e [18]. Para analisar os resultados obtidos por cada estratégia, consideramos a métrica de retorno acumulado dado por

$$
R_{T}=\prod_{t=1}^{T}\left(1+\hat{r_{t}}\right)-1
$$

onde $\hat{r}_{t}$ é o retorno observado do portfólio no tempo $t$, e como métricas de performance, utilizamos o índice de $\operatorname{Sharpe}^{4}\left(I S_{p}\right)$ do portfólio

$$
I S_{p}=\frac{r_{p}-r_{f}}{\sigma_{p}}
$$

onde $r_{p}=\left(1+R_{T}\right)^{\frac{252}{T}}-1$, que é retorno efetivo anualizado do portfólio, $\sigma_{p}$ é o desvio padrão ${ }^{5}$ dos retornos anuais do portfólio, $r_{f}^{6}$ é a taxa livre de risco ao ano.

${ }^{1}$ Julia for Mathematical Optimization, que é um domínio específico para modelagem matemática, embutido dentro da programação Julia.

${ }^{2}$ Estratégia Benchmark é a referência contra a qual o desempenho da nossa estratégia será comparada.

${ }^{3}$ Naive (ingênua), pois o tomador de decisão não utiliza algum modelo mais elaborado de alocação, apenas aloca igualmente em cada um dos ativos disponíveis, independentemente do risco de cada ativo.

${ }^{4} \mathrm{O} I S$ é uma medida de performance que foi desenvolvido pelo economista, laureado pelo Prêmio Nóbel de Economia, Willian F. Sharpe, que mensura o retorno médio em excesso, relativo à taxa livre de risco, por unidade de volatilidade.

${ }^{5}$ Calculamos o desvio padrão dos retorno gerados em uma janela rolante de 252 dias úteis, deslocando a janela a cada um dia útil.

${ }^{6}$ Utilizamos a taxa anual do título do tesouro americano de 1 mês. A taxa foi extraída da carteira Fama/French 3 Factors [Daily], na aba U.S. Research Returns Data. 
Também calculamos o máximo drawdown $\left(M D D_{T}\right)$ da carteira, o qual podemos visualizar mais facilmente na Figura 4.1.

$$
M D D_{T}=\min _{\tau \in[0, T]}\left\{\min _{t \in[0, \tau]}\left[0, \frac{R_{\tau}}{R_{t}}-1\right]\right\}
$$

onde $M D D_{T}$ corresponde a maior perda percentual do portfólio investido em um determinado período.

Figura 4.1: Máximo Draw Down

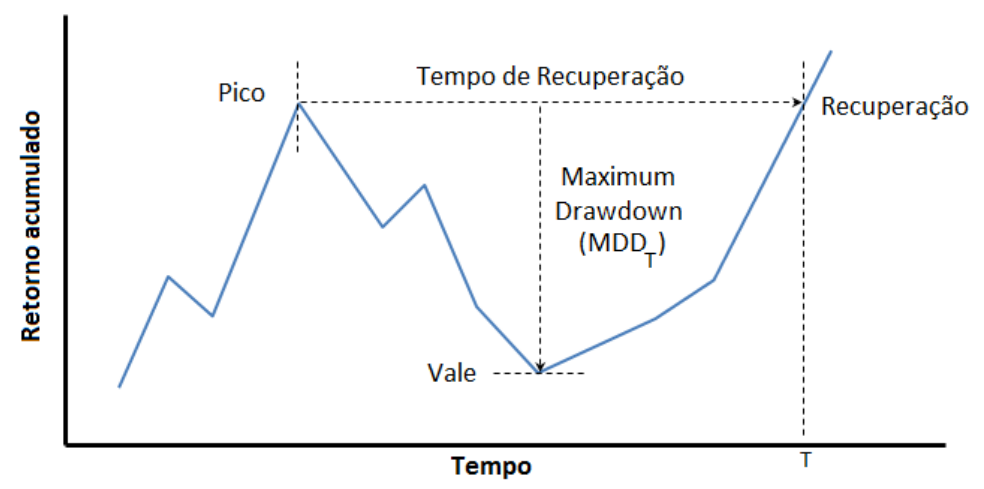

O MDD é a distância entre o maior pico e o menor vale subsequente dado um período específico.

Avaliamos o desempenho do portfólios ótimos contra as estratégias benchmark, realizando um backtest dos modelos $C h u l l_{N}$, do investimento passivo do índice SP500 e da estratégia naive da carteira $1 / \mathrm{d}$. Basicamente simulamos as diferentes estratégias nos dados históricos e avaliamos como os modelos se comportaram.

A tabela 4.1 apresenta o resultado comparativo para diferentes amostras $\Theta_{N}$ para um período de 20 anos. Na segunda coluna estão os retornos acumulados por cada carteira em todo período analisado, na terceira coluna os retornos anualizados, a quarta coluna mostra o desvio padrão dos retornos anuais e a última coluna mostra o tempo computacional, em segundos, utilizados para definir a estratégia à cada dia. 
Tabela 4.1: Tabela comparativa da estratégia Chull $_{N}$, com 2 ativos, para diferentes especificações. Amostra entre 20/03/1998 e 29/03/2018, totalizando 5040 observações.

\begin{tabular}{lcccccc}
\hline Estratégia & $\mathbf{R}_{\mathrm{T}}$ & $\mathbf{r}_{\mathrm{p}}$ & $\boldsymbol{\sigma}_{\mathrm{p}}$ & $\mathbf{I S}_{\mathrm{p}}$ & $\mathbf{M D D}_{\mathrm{T}}$ & $\begin{array}{c}\text { Tempo } \\
\text { Computacional }\end{array}$ \\
\hline Chull $_{5}$ & $360.31 \%$ & $7.93 \%$ & $17.14 \%$ & 0.38 & $-55.47 \%$ & 0.006 \\
Chull $_{10}$ & $504.48 \%$ & $9.41 \%$ & $15.00 \%$ & 0.53 & $-46.66 \%$ & 0.014 \\
Chull $_{15}$ & $457.96 \%$ & $8.98 \%$ & $14.56 \%$ & 0.52 & $-43.65 \%$ & 0.023 \\
Chull $_{30}$ & $392.54 \%$ & $8.30 \%$ & $14.65 \%$ & 0.47 & $-46.39 \%$ & 0.090 \\
Chull $_{50}$ & $356.82 \%$ & $7.89 \%$ & $15.10 \%$ & 0.43 & $-46.69 \%$ & 0.176 \\
Chull $_{100}$ & $344.19 \%$ & $7.74 \%$ & $15.33 \%$ & 0.41 & $-50.46 \%$ & 1.091 \\
Chull $_{252}$ & $333.23 \%$ & $7.61 \%$ & $14.79 \%$ & 0.42 & $-45.92 \%$ & 4.840 \\
Chull $_{350}$ & $455.48 \%$ & $8.95 \%$ & $17.14 \%$ & 0.44 & $-39.56 \%$ & 9.277 \\
Chull $_{400}$ & $475.32 \%$ & $9.14 \%$ & $14.16 \%$ & 0.54 & $-43.20 \%$ & 12.355 \\
Chull $_{450}$ & $416.99 \%$ & $8.56 \%$ & $13.77 \%$ & 0.52 & $-43.20 \%$ & 15.208 \\
Chull $_{500}$ & $431.46 \%$ & $8.71 \%$ & $13.79 \%$ & 0.53 & $-43.20 \%$ & 19.692 \\
Chull $_{600}$ & $444.92 \%$ & $8.85 \%$ & $13.76 \%$ & 0.54 & $-43.20 \%$ & 30.369 \\
\hline Carteira 1/d & $409.09 \%$ & $8.48 \%$ & $14.27 \%$ & 0.49 & $-46.54 \%$ & - \\
SP500 & $141.81 \%$ & $4.51 \%$ & $16.74 \%$ & 0.18 & $-56.78 \%$ & - \\
\hline
\end{tabular}

Notamos na tabela 4.1 que sete das carteiras que utilizaram a estratégia proposta apresentaram retorno acumulado superior aos respectivos benchmarks, em especial a $\mathrm{Chull}_{10}$, que teve o melhor desempenho dentre todas as carteiras. Porém, percebemos uma maior variação no desempenho das carteiras que utilizaram tamanhos de amostras $\Theta_{N}$ menores, onde inicialmente identificou-se a tendência de redução no desempenho, ao passo que aumentamos o tamanho da amostra. Logo em seguida percebemos não somente uma retomada no ganho de performance na carteira $\mathrm{Chull}_{350}$, mas também nota-se que o desempenho das demais carteiras oscilam em níveis muito próximos. Quando levamos em consideração o Índice de Sharpe e o máximo drawdown, a carteira $C h u l l_{400}$ foi a que apresentou a melhor relação entre o risco e retorno dentre as demais carteiras.

A Figura 4.2 mostra gráfico da evolução da performance acumulada de todas as estratégias Chull contra a carteira $1 / d$ e o índice SP500. 
Figura 4.2: Evolução da performance Acumulada (\%) de cada uma das carteiras com 2 ativos contra os respectivos Benchmarks entre 20 de março de 1998 e 29 de março de 2018.
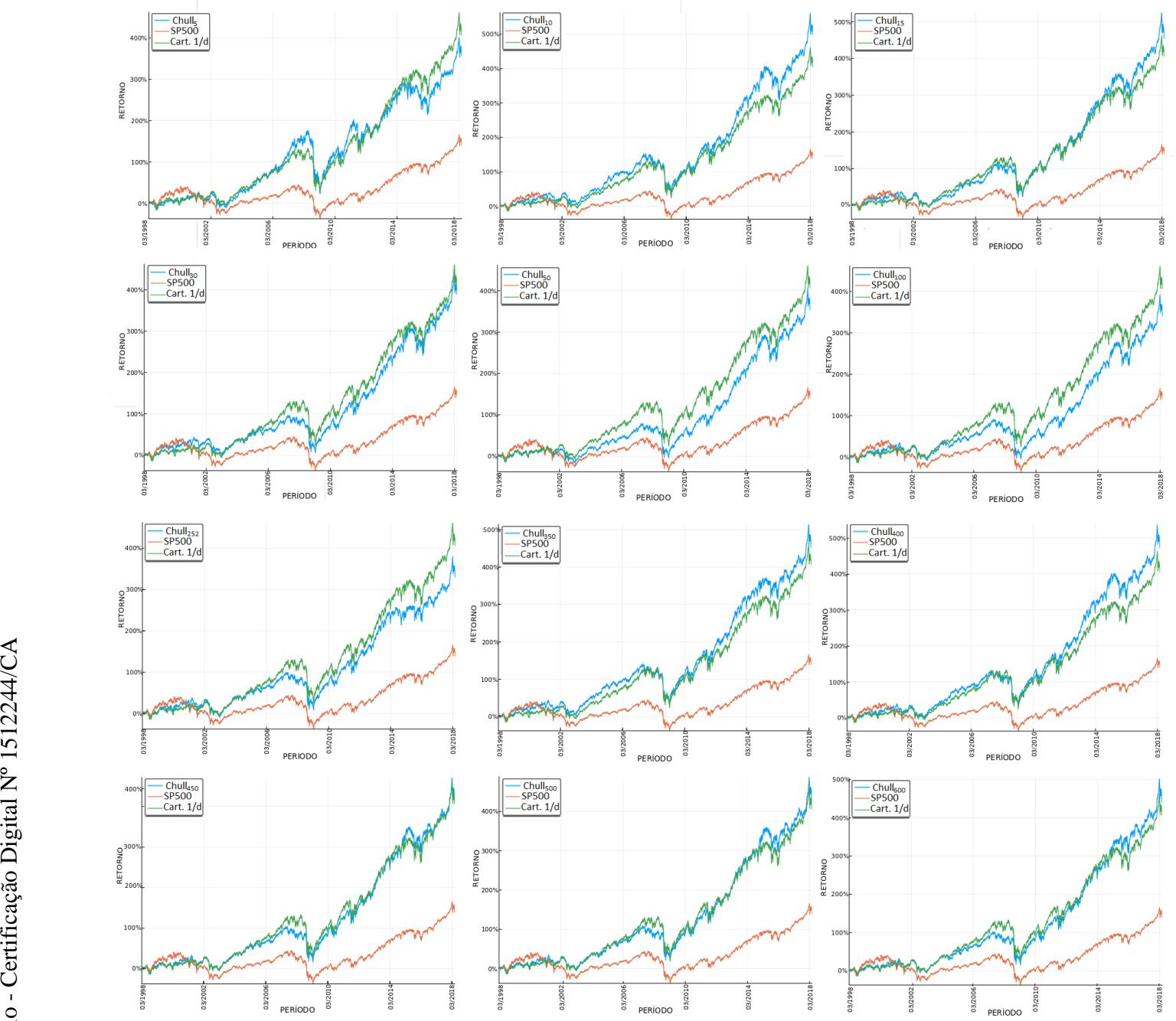

As estratégias Chull $_{10}$, Chull $_{350}$ e Chull $_{400}$ foram as que apresentaram performances mais consistentes em relação à todas as outras. No entanto, a primeira foi a única que foi estocasticamente dominante em relação a carteira $1 / \mathrm{d}$ durante o período analisado, enquanto a segunda acumulou performance superior até 2018, porém durante o período de crise gerou perdas que a fez empatar com a carteira $1 /$ d. Em 2009, a $C_{\text {Chull }} 350$ iniciou uma rápida recuperação que possibilitou uma retorno acumulado aproximadamente $11.5 \%$ maior que o benchmark. A terceira carteira manteve até o final de 2011 um comportamento muito próximo da estratégia $1 / \mathrm{d}$ e, a partir deste ano, melhorou sua performance, descolando a diferença do desempenho até o final do período analisado.

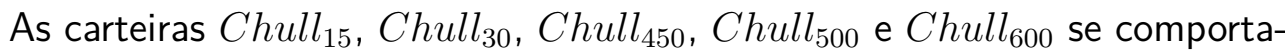
ram muito próximos a carteira $1 / \mathrm{d}$ em praticamente todo o período observado, e 
tal comportamento, foi ainda mais parecido entre os anos de 2007 e 2008, período que deflagrou a crise do subprime.

Durante os primeiros três anos do período analisado, o índice SP500 foi superior a todas as estratégias, porém o índice permaneceu de lado ${ }^{7}$ por quase 12 anos, tornado a estratégia passiva a pior entre todas.

A Figura 4.3 mostra a relação entre risco e retorno oferecido pela estratégia sugeridas por este trabalho. O eixo vertical é retorno diário médio dos dias que a estratégia proposta foi implementada e no eixo horizontal, a volatilidade ${ }^{8}$ dos retornos diários dos portfólios.

Figura 4.3: Relação entre Risco e Retorno das estratégias entre 20 de março de 1998 e 29 de março de 2018.

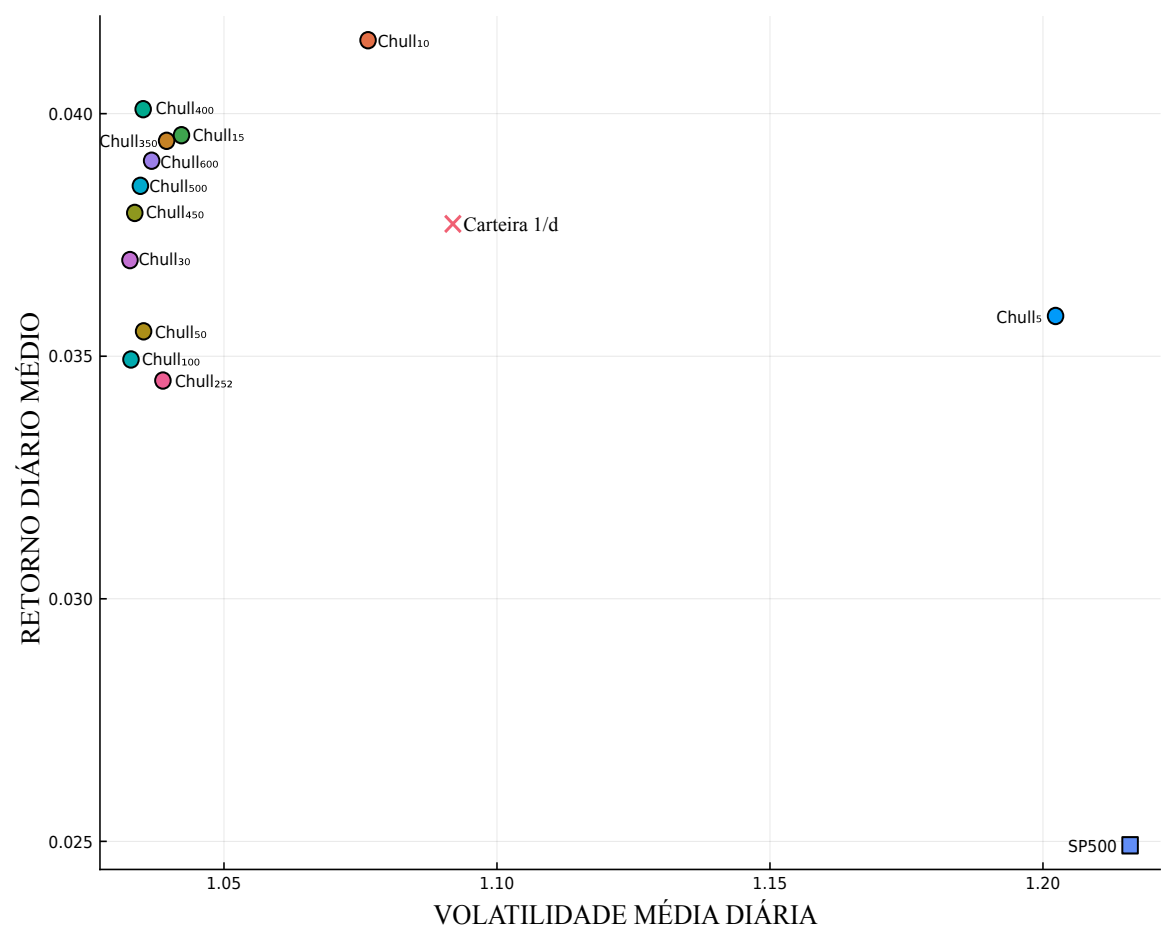

Notamos uma boa relação entre o risco e o retorno das estratégias Chull em relação aos benchmarks. Apesar das carteiras Chull $_{30}$, Chull $_{50}$, Chull $_{100}$ e $\mathrm{Chull}_{252}$ terem gerado retornos acumulados inferiores a carteira $1 / \mathrm{d}$, elas compensam com um risco, representado pela volatilidade diária, significativamente menor que o benchmark. Contudo as estratégias Chull $_{10}, C_{\text {Chll }}$, Chull $_{350}$,

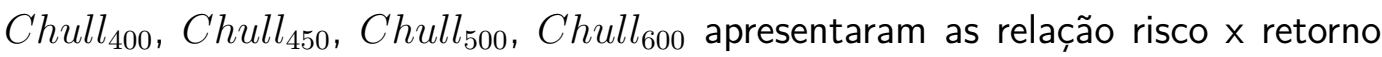
mais atrativas em comparação a carteira igualitária, pois geraram uma rentabilidade superior com o mesmo nível de volatilidade. Em relação a análise da Figura 4.3,

${ }^{7} \mathrm{~A}$ expressão "O mercado está de de lado" significa que o preço das ações valorizam e desvalorizam em pequenas proporções ao longo do tempo, fazendo com que o índice de ações oscile dentro de um intervalo de preço por um longo período.

${ }^{8}$ Utilizamos o desvio padrão como métrica de risco, que é um dos mais usados em aplicações. 
as carteiras $\mathrm{Chull}_{10}$ e $C h u l l_{400}$ são as mais atrativas, e dependendo de qual fator seja mais importante para o tomador e decisão, volatilidade ou rentabilidade média, qualquer uma das duas estratégias pode ser considerada a melhor, e com isto, uma opção mais vantajosa que a carteira $1 / \mathrm{d}$. A estratégia passiva, que investe no índice SP500 resultou tanto no menor rendimento quando no maior risco.

Para checar a qualidade do nosso modelo em diferentes períodos em relação a amostra, consideramos que o investidor mantenha a estratégia por diferentes intervalos de tempo. A Figura 4.4 mostra o Trailing dos retornos da nossa estratégia que melhor performou na amostra analisada $\left(\right.$ Chull $\left._{10}\right)$ para vários intervalos de tempo junto com o da carteira $1 / \mathrm{d}$. O trailing das outras carteiras estão no apêndice. $O$ trailing ${ }^{9}$ dos retornos exibe praticamente e mesma frequência de rendimentos positivos que o benchmark, alem disso observamos que a nossa estratégia aumenta este comportamento quando ampliamos o intervalo de tempo de manutenção considerado.

Figura 4.4: Trailing de retornos do modelo Chull $_{10}$ e da carteira 1/d para vários intervalos (6 meses, 1 aqnos, 2 anos e 3 anos), amostra de 5040 observações diários entre os dias 20 de março de 1998 e 29 de março de 2018

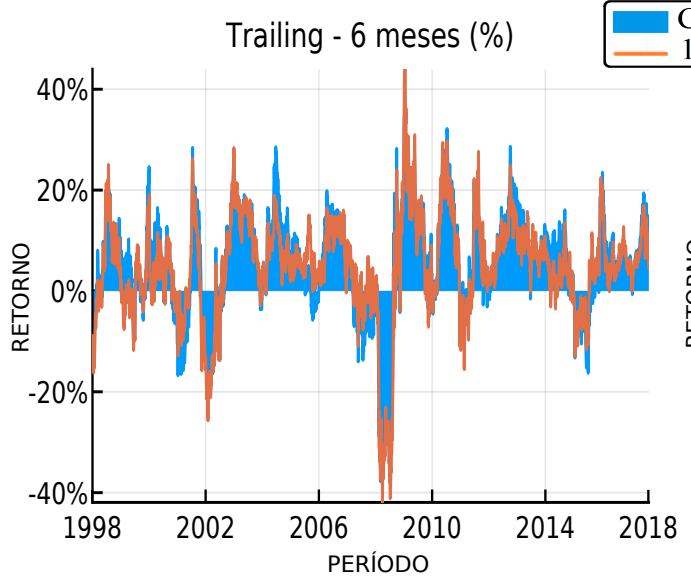

Trailing - 2 anos (\%)
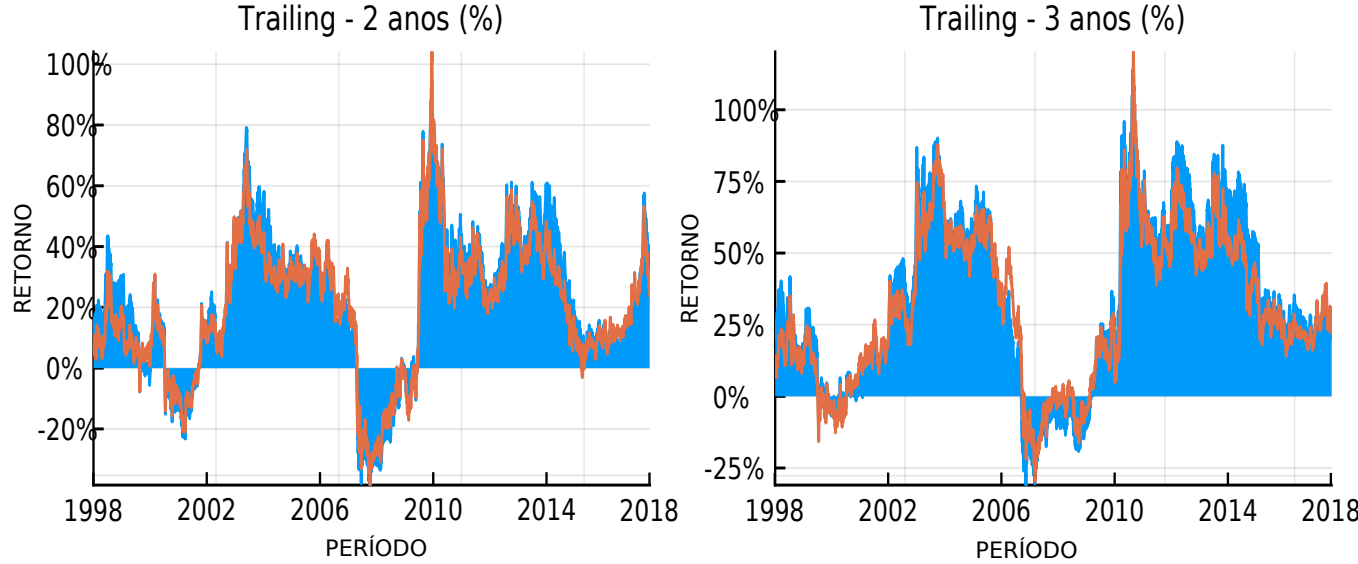

${ }^{9}$ Retorno observado de uma ativo em um determinado período de tempo. 
Na Figura 4.4, notamos que as duas estratégias possuem uma correlação significativa entre elas, pois ambas as carteiras apresentam retornos positivos e negativos simultaneamente em praticamente todos os períodos, e quando uma carteira apresenta um resultado mais extremo, a outra também apresenta. Contudo, nota-se que o modelo Chull $_{10}$ gerou, na maiorias das vezes, resultados positivos maiores e negativos menores, que a carteira $1 / \mathrm{d}$. Este fato pode ser melhor verificado na Tabela A.2, que mostra a proporção de dias em que cada estratégia gerou um retorno superior a carteira $1 / \mathrm{d}$. Nesta tabela, verificamos que todas as estratégias Chull geraram na maioria dos dias retornos inferiores a carteira igualitária, indicando que as carteiras mais rentáveis "perdem" por pouco, mas "ganham" por uma diferença mais relevante.

$\mathrm{Na}$ Tabela 4.2, podemos observar o resultado exposto na figura 4.4 de uma outra perspectiva, pois mostra a proporção de retornos positivos e negativos observados nas janelas rolantes e períodos analisados no trailing da figura 4.4, para todas as carteiras.

Tabela 4.2: Proporção (\%) dos retornos positivos e negativos observados de cada estratégia com 2 ativos, em diferentes intervalos de tempo do traling em relação a amostra que 20 de março de 1998 e 29 de março de 2018.

\begin{tabular}{|c|c|c|c|c|c|c|c|c|}
\hline \multirow{3}{*}{ Estratégias } & \multicolumn{2}{|c|}{6 meses } & \multicolumn{2}{|c|}{1 ano } & \multicolumn{2}{|c|}{2 anos } & \multicolumn{2}{|c|}{3 anos } \\
\hline & \multicolumn{2}{|c|}{ \% Prop. dos Retornos } & \multicolumn{2}{|c|}{ \% Prop. dos Retornos } & \multicolumn{2}{|c|}{ \% Prop. dos Retornos } & \multicolumn{2}{|c|}{ \% Prop. dos Retornos } \\
\hline & Positivos & Negativos & Positivos & Negativos & Positivos & Negativos & Positivos & Negativos \\
\hline Chull $_{5}$ & $73.25 \%$ & $26.75 \%$ & $76.69 \%$ & $23.31 \%$ & $77.97 \%$ & $22.03 \%$ & $83.40 \%$ & $16.60 \%$ \\
\hline Chull $_{10}$ & $74.13 \%$ & $25.87 \%$ & $79.09 \%$ & $20.91 \%$ & $82.56 \%$ & $17.44 \%$ & $83.47 \%$ & $16.53 \%$ \\
\hline Chull $_{15}$ & $76.12 \%$ & $23.88 \%$ & $79.67 \%$ & $20.33 \%$ & $81.96 \%$ & $18.04 \%$ & $82.56 \%$ & $17.44 \%$ \\
\hline Chull $_{30}$ & $74.29 \%$ & $25.71 \%$ & $79.30 \%$ & $20.70 \%$ & $81.96 \%$ & $18.04 \%$ & $79.06 \%$ & $20.94 \%$ \\
\hline Chull $_{50}$ & $72.03 \%$ & $27.97 \%$ & $77.40 \%$ & $22.60 \%$ & $81.48 \%$ & $18.52 \%$ & $78.61 \%$ & $21.39 \%$ \\
\hline Chull $_{100}$ & $72.81 \%$ & $27.19 \%$ & $78.88 \%$ & $21.12 \%$ & $81.23 \%$ & $18.77 \%$ & $78.50 \%$ & $21.50 \%$ \\
\hline Chull $_{252}$ & $71.48 \%$ & $28.52 \%$ & $79.38 \%$ & $20.62 \%$ & $83.40 \%$ & $16.60 \%$ & $80.32 \%$ & $19.68 \%$ \\
\hline Chull $_{350}$ & $74.96 \%$ & $25.04 \%$ & $80.49 \%$ & $19.51 \%$ & $83.31 \%$ & $16.69 \%$ & $89.35 \%$ & $10.65 \%$ \\
\hline Chull $_{400}$ & $76.31 \%$ & $23.69 \%$ & $78.71 \%$ & $21.29 \%$ & $86.06 \%$ & $13.94 \%$ & $85.13 \%$ & $14.87 \%$ \\
\hline Chull $_{450}$ & $75.21 \%$ & $24.79 \%$ & $78.40 \%$ & $21.60 \%$ & $85.91 \%$ & $14.09 \%$ & $84.54 \%$ & $15.46 \%$ \\
\hline Chull $_{500}$ & $74.35 \%$ & $25.65 \%$ & $78.98 \%$ & $21.02 \%$ & $85.95 \%$ & $14.05 \%$ & $84.54 \%$ & $15.46 \%$ \\
\hline Chull $_{600}$ & $75.05 \%$ & $24.95 \%$ & $80.07 \%$ & $19.93 \%$ & $85.84 \%$ & $14.16 \%$ & $84.26 \%$ & $15.74 \%$ \\
\hline Carteira $1 / \mathrm{d}$ & $75.68 \%$ & $24.32 \%$ & $81.52 \%$ & $18.48 \%$ & $82.98 \%$ & $17.02 \%$ & $85.53 \%$ & $14.47 \%$ \\
\hline SP500 & $69.01 \%$ & $30.99 \%$ & $73.22 \%$ & $26.78 \%$ & $71.36 \%$ & $28.64 \%$ & $66.08 \%$ & $33.92 \%$ \\
\hline
\end{tabular}

Apesar da estratégia $\mathrm{Chull}_{10}$ possuir a maior rentabilidade acumulada, a carteira esteve entre as piores em relação a proporção de retornos positivos em todas as janelas de tempo observadas. Somente na análise de intervalos de um ano, que todas as estratégias Chull perderam simultaneamente para a carteira $1 /$ d. Dentre todas as estratégias, a $C h u l l_{400}$ foi a mais competitiva, superando o benchmark em praticamente todos os cenários, seguida pela Chull ${ }_{15}$ que performou muito próximo da carteira $1 / \mathrm{d}$. Porem, ao analisarmos o ganho de performance, isto é, o aumento na proporção do número de períodos com retornos positivos em cada intervalo de tempo analisado do traling, as estratégias Chull 
com $N=\{5,10,15,350\}$ foram as únicas que obtiveram ganho de performance à cada aumento de intervalo. A carteira $\mathrm{Chull}_{350}$ foi a que gerou o maior aumento na proporção de retorno positivos, num total de $19.19 \%$ entre os períodos de 6 meses e 3 anos, seguido pelas carteiras Chull $_{5}$ e $C h u l l_{500}$, que melhoraram em $13.85 \%$ e $13.70 \%$ respectivamente.

As informações obtidas até o momento indicam que apesar da estratégia proposta gerar uma maior frequência de perdas que a carteira $1 / d$, as carteiras tendem a compensar com rendimentos de maior magnitude, fato que permitiu grande parte das carteiras superarem o benchmark. Para analisarmos mais detaIhadamente este fato, a Tabela 4.3 mostra a proporção de dias, dentre os 5040 observados, que as estratégias geraram retornos positivos (primeira coluna), como também a proporção de dias que as carteiras realizaram retornos maiores que a carteira igualitária e o índice SP500.

Tabela 4.3: Tabela com a proporção de dias que as estratégias geraram rendimentos positivos e a proporção de dias que superaram as carteira 1/d e o índice SP500 em relação ao retorno gerado.

\begin{tabular}{|c|c|c|c|}
\hline \multirow{2}{*}{ Estratégias } & \multirow{2}{*}{$\begin{array}{l}\text { \%Prop. dos } \\
\text { Dias com } \\
\text { Retorno } \\
\text { Positivo }\end{array}$} & \multicolumn{2}{|c|}{$\begin{array}{l}\text { \% Prop. Dias que o Retorno } \\
\text { da Estratégia foi Superior a }\end{array}$} \\
\hline & & Carteira 1/d & SP500 \\
\hline $\mathrm{Chull}_{5}$ & $53.55 \%$ & $48.43 \%$ & $49.56 \%$ \\
\hline $\mathrm{Chull}_{10}$ & $53.83 \%$ & $48.29 \%$ & $50.22 \%$ \\
\hline $\mathrm{Chull}_{15}$ & $53.65 \%$ & $48.21 \%$ & $49.80 \%$ \\
\hline $\mathrm{Chull}_{30}$ & $54.01 \%$ & $48.25 \%$ & $49.60 \%$ \\
\hline $\mathrm{Chull}_{50}$ & $53.83 \%$ & $47.82 \%$ & $49.60 \%$ \\
\hline Chull $_{100}$ & $54.11 \%$ & $47.94 \%$ & $49.33 \%$ \\
\hline Chull $_{252}$ & $54.03 \%$ & $48.06 \%$ & $49.33 \%$ \\
\hline Chull $_{350}$ & $54.38 \%$ & $48.27 \%$ & $49.68 \%$ \\
\hline Chull $_{400}$ & $54.31 \%$ & $48.25 \%$ & $49.66 \%$ \\
\hline Chull $_{450}$ & $54.25 \%$ & $48.31 \%$ & $49.62 \%$ \\
\hline Chull $_{500}$ & $54.13 \%$ & $48.29 \%$ & $49.46 \%$ \\
\hline Chull $_{600}$ & $54.13 \%$ & $48.41 \%$ & $49.50 \%$ \\
\hline Carteira 1/d & $54.44 \%$ & - & $48.97 \%$ \\
\hline SP500 & $53.27 \%$ & $51.03 \%$ & - \\
\hline
\end{tabular}

Notamos que todas as carteiras geraram proporções muito próximas de dias com rendimentos positivos, com uma diferença entre a maior e menor de aproximadamente $1.17 \%$ entre elas. Comparando o resultado diário de cada carteira, todas as estratégias Chull, inclusive as que tiveram maior retorno acumulado, obtiveram uma maior frequência de dias com retornos inferiores que os gerados pelos benchmarks, exceto a Chull $_{10}$ com o SP500.

Em seguida mostraremos como se comportaram algumas das carteira com 2 ativos em relação a ao percentual da riqueza total investido em cada um dos 
ativos disponíveis. $\mathrm{O}$ ativo 2 foi o que apresentou maior volatilidade, como também rendimento no período analisado.

Figura 4.5: Alocação percentual em relação a riqueza total das carteiras Chull $_{5}$,

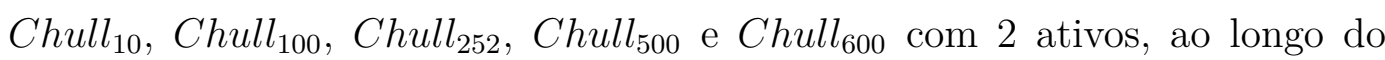
tempo, entre os dias 20 de março 1998 e 29 de março de 2018.
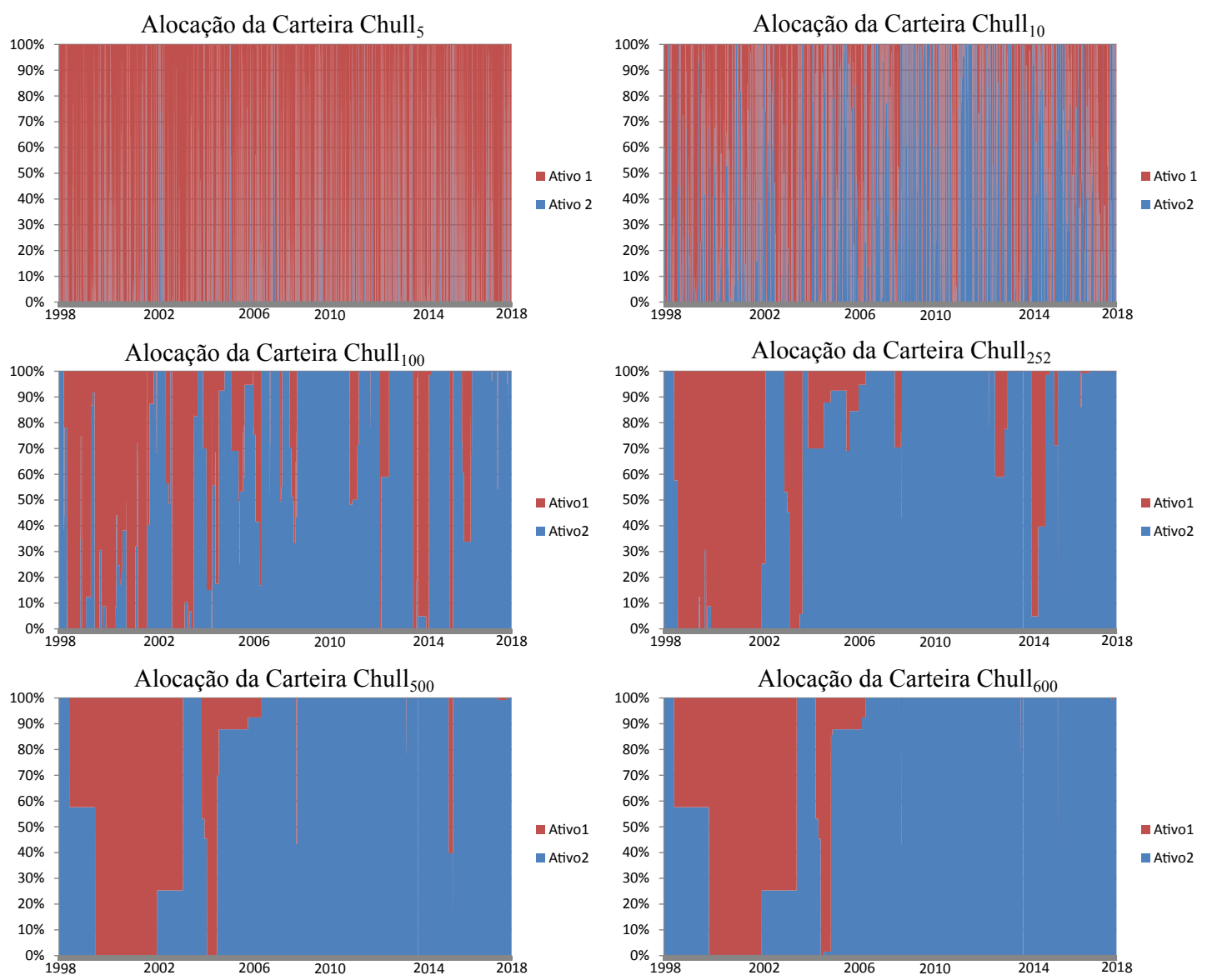

Na Figura 4.5 mostra que o modelo apresenta um comportamento cada vez mais passivo em relação a alocação da riqueza, isto é, efetua um número cada vez menor de mudanças no percentual da riqueza alocado em cada ativo, quando aumentamos a janela de tempo observada para elaboração das estratégias. Além disto, também apresenta uma preferência pelo ativo com maior volatilidade.

Para investigar como o nosso modelo se comporta durante o processo de tomada decisão em diferentes condições do mercado, a figura 4.6 mostra a dispersão do retorno dos ativos em diferentes condições de mercado, sendo eles um período de crise (setembro, outubro de novembro de 2008) e um período de não crise (setembro, outubro e novembro de 2017). A figura também mostra os conjuntos de incerteza dos parâmetros, que foram construídos com os dados observados em cada período, e os intervalos de confiança, definido em (2.4). Nesta 
figura estão os retornos diários dos ativos da carteira Industry Portfólio com dois ativos.

Figura 4.6: Gráfico de dispersão do retorno dos ativos arriscados, seus respectivos conjuntos de incerteza e intervalos de confiança: Portfólio Industry com 2 ativos em diferentes condições de mercado.
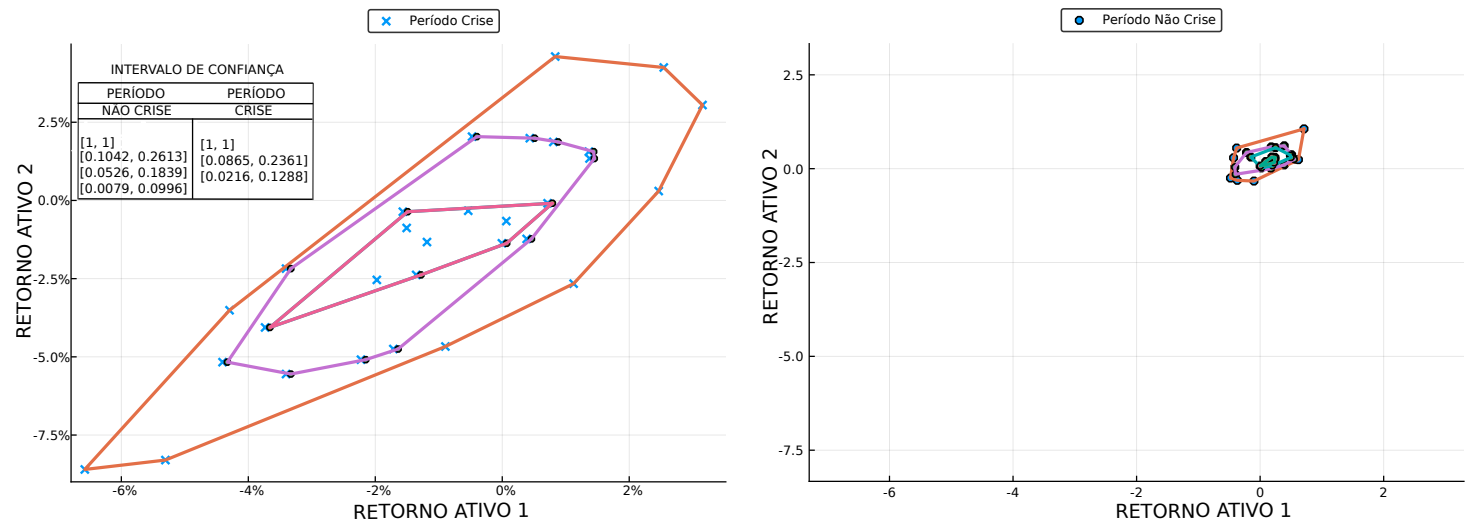

Notamos na Figura 4.6 a ocorrência de retornos extremos durante o período de crise, o que gera conjuntos de incerteza dos parâmetros aleatórios bem maiores que os formados em períodos fora de crise. O formato dos conjuntos no período de não crise sugere uma baixa correlação dos retornos, indicando que a formação do preço de cada ativos é uma função dos fatores de risco do mercado ${ }^{10}$, mas principalmente de fatores de risco específicos ${ }^{11}$ de cada empresa. Já o formato do conjunto no período de crise fornece evidências da convergência da correlação dos retornos para um, onde todos os ativos se desvalorizam/valorizam concomitantemente, sugerindo que os fatores de mercado têm maior relevância na formação do preço dos ativos.

Para investigarmos um pouco mais o comportamento exibido na Figura 4.6, também construímos os fechos convexos de outra carteira com dois ativos arriscados, que investe nos índices Down Jones e SP500, para os períodos de crise e não crise, apresentado na Figura 4.7.

\footnotetext{
${ }^{10}$ São características da economia como um todo, que afetam todos os participantes do mercado, como colapso do sistema financeiro, grandes variações na taxa de juros e câmbio, etc.

${ }^{11}$ São características específicas da empresa, como setor de atuação, fatores operacionais, qualidade da governança corporativa, entre outros, que afetam a avaliação da empresa pelos agentes financeiros e consequentemente o preço da ação.
} 
Figura 4.7: Gráfico de dispersão do retorno da carteira de 2 ativos, formada com os índices SP500 e Down Jones, seus respectivos conjuntos de incerteza.

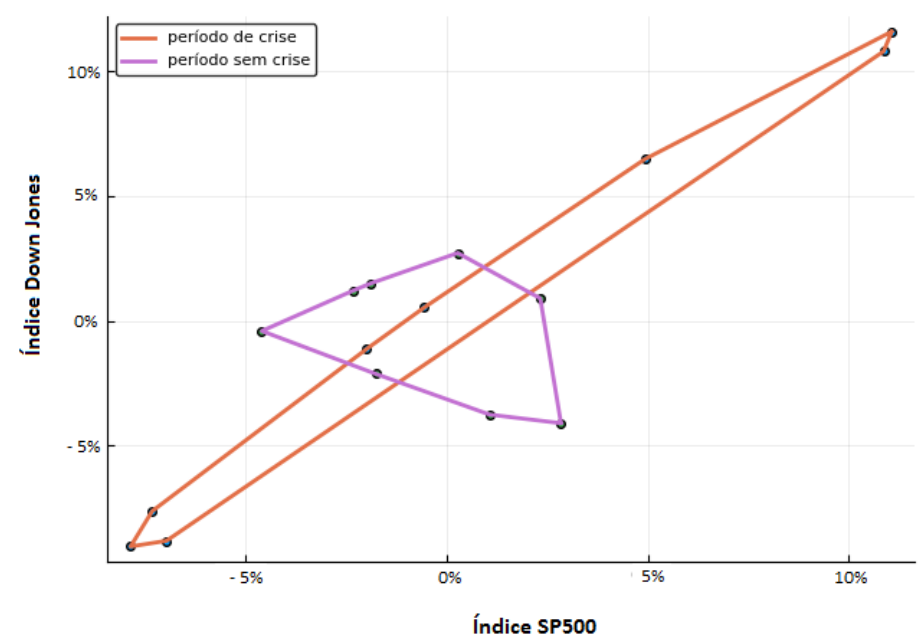

Notamos uma correlação ainda maior entre os dois índices, pois no período de crise o conjunto formado é ainda mais achatado, mostrando que os ativos assumem praticamente os mesmos valores, enquanto no período de menor volatilidade (não crise) é formado um conjunto com elementos distribuídos mais uniformemente em torno do ponto com zero em todas as coordenadas.

Para termos uma avaliação mais minuciosa do desemprenho da nosso algorítimo, realizamos o backtest mais carteiras, porém com 5, 10, 17 e 30 ativos, que foram elaboradas com os mesmos tamanhos de amostra $\Theta$ utilizados na elaboração das carteira com 2 ativos e, comparamos o desemprenho gerado entre elas. A figura 4.4 mostra a proporção total de dias que todas as carteiras a estratégia proposta, com carteiras compostas com 2, 5, 10, 17 e 30 ativos, geraram retornos positivos. No Apêndice colocamos tabelas com resultados mais detalhados de cada carteira. 
Tabela 4.4: Tabela comparativa com a proporção de dias que houve retorno positivo carteiras com 2, 5, 10, 17 e 30 ativos.

\begin{tabular}{cccccc}
\hline \multicolumn{5}{c}{ PROPORÇÃO DE DIAS COM RETORNOS POSITIVOS } \\
Estratégias & 2 ATIVO & 5 ATIVOS & 10 ATIVO & 17 ATIVOS & 30 ATIVOS \\
\hline Chull $_{5}$ & $53.54 \%$ & $53.60 \%$ & $53.32 \%$ & $53.24 \%$ & $53.46 \%$ \\
Chull $_{10}$ & $53.82 \%$ & $54.16 \%$ & $53.62 \%$ & $54.14 \%$ & $53.32 \%$ \\
Chull $_{15}$ & $53.64 \%$ & $53.90 \%$ & $53.66 \%$ & $54.26 \%$ & $53.01 \%$ \\
Chull $_{30}$ & $54.00 \%$ & $53.94 \%$ & $53.86 \%$ & $53.82 \%$ & $53.74 \%$ \\
Chull $_{50}$ & $53.82 \%$ & $53.17 \%$ & $53.58 \%$ & $53.62 \%$ & $52.95 \%$ \\
Chull $_{100}$ & $54.10 \%$ & $53.66 \%$ & $53.60 \%$ & $53.38 \%$ & $52.53 \%$ \\
Chull $_{252}$ & $54.02 \%$ & $54.16 \%$ & $53.84 \%$ & $53.86 \%$ & $53.09 \%$ \\
Chull $_{350}$ & $54.38 \%$ & $53.90 \%$ & $53.92 \%$ & $53.84 \%$ & $53.90 \%$ \\
Chull $_{400}$ & $54.30 \%$ & $54.16 \%$ & $54.22 \%$ & $54.38 \%$ & $54.26 \%$ \\
Chull $_{450}$ & $54.25 \%$ & $54.14 \%$ & $53.86 \%$ & $53.30 \%$ & $54.02 \%$ \\
Chull $_{500}$ & $54.13 \%$ & $53.72 \%$ & $53.66 \%$ & $53.62 \%$ & $54.00 \%$ \\
Chull $_{600}$ & $54.13 \%$ & $54.20 \%$ & $53.38 \%$ & $53.52 \%$ & $54.24 \%$ \\
\hline Cart. 1/d $^{54.44 \%}$ & $53.99 \%$ & $54.35 \%$ & $53.93 \%$ & $54.05 \%$ \\
\hline
\end{tabular}

Não identificamos alguma mudança no comportamento das carteiras ao analisar a Tabela 4.4. Praticamente não houve alteração nas proporções de dias com retornos positivos ao passo que aumentamos o número de ativos, pois todas as carteiras tiveram um comportamento muito similar, oscilando esta proporção em cada alteração de tamanho da amostra $\Theta_{N}$ que analisamos. A carteira Chull $_{400}$ foi a que gerou maior frequência, inclusive superando estratégia naive na maioria das carteiras com diferentes números de ativos, sendo estas duas as únicas que tiveram uma frequência média maior que 54\%. Apesar do comportamento estático em relação à proporção dos dias com rendimento positivo identificado na Tabela 4.4, as Tabelas A.3 e A.4, mostram que o aumento para 5 ativos foi quanto o modelo apresentou o maior aumento geral na performance de todas as carteiras, tanto que a carteira $1 / \mathrm{d}$ gerou o segundo pior desempenho, ficando à frente somente da Chull $_{5}$. Em relação a variação do número de ativos, reparamos, ao passo que aumentamos o tamanho das carteiras, um ganho de performance nas estratégias Chull $_{N}$ elaboradas com amostas $\Theta_{N}$ maiores e perda de performance nas carteiras com amostras menores. Em especial, temos Chull $_{500}$, que foi a carteira mais consistente, tendo em vista todas as métricas utilizadas.

Logo em seguida, apresentamos uma tabela comparativa com o desvip padrão dos retornos diários de cada uma das carteiras analisadas. 
Tabela 4.5: Tabela comparativa da volatilidade dos retornos diários das carteiras com 2, 5, 10, 17 e 30 ativos.

$$
\text { DESVIO PADRÃO DOS RETORNOS DIÁRIOS }
$$

Estratégias 2 ATIVOS 5 ATIVOS 10 ATIVOS 17 ATIVOS 30 ATIVOS

\begin{tabular}{llllll}
\hline Chull $_{5}$ & $1.202 \%$ & $1.115 \%$ & $1.097 \%$ & $1.172 \%$ & $1.250 \%$ \\
Chull $_{10}$ & $1.076 \%$ & $1.036 \%$ & $0.998 \%$ & $1.004 \%$ & $1.021 \%$ \\
Chull $_{15}$ & $1.042 \%$ & $1.030 \%$ & $0.977 \%$ & $0.973 \%$ & $0.974 \%$ \\
Chull $_{30}$ & $1.033 \%$ & $1.017 \%$ & $0.9534 \%$ & $0.945 \%$ & $0.942 \%$ \\
Chull $_{50}$ & $1.035 \%$ & $1.019 \%$ & $0.947 \%$ & $0.938 \%$ & $0.932 \%$ \\
Chull $_{100}$ & $1.033 \%$ & $1.010 \%$ & $0.943 \%$ & $0.936 \%$ & $0.925 \%$ \\
Chull $_{252}$ & $1.039 \%$ & $1.023 \%$ & $0.953 \%$ & $0.968 \%$ & $0.935 \%$ \\
Chull $_{350}$ & $1.039 \%$ & $1.021 \%$ & $0.955 \%$ & $0.963 \%$ & $0.943 \%$ \\
Chull $_{400}$ & $1.035 \%$ & $1.020 \%$ & $0.962 \%$ & $0.964 \%$ & $0.947 \%$ \\
Chull $_{450}$ & $1.034 \%$ & $1.022 \%$ & $0.966 \%$ & $0.966 \%$ & $0.950 \%$ \\
Chull $_{500}$ & $1.035 \%$ & $1.027 \%$ & $0.969 \%$ & $0.969 \%$ & $0.956 \%$ \\
Chull $_{600}$ & $1.037 \%$ & $1.031 \%$ & $0.982 \%$ & $0.968 \%$ & $0.961 \%$ \\
\hline Cart. $1 / \mathrm{d}$ & $1.092 \%$ & $1.163 \%$ & $1.138 \%$ & $1.236 \%$ & $1.213 \%$ \\
\hline
\end{tabular}

Podemos perceber na Tabela 4.5 uma diminuição do risco diário da carteira, mensurado através da sua volatilidade, quando aumentamos o número de ativos, dado que fixamos o o tamanho na amostra $\Theta_{N}$. O mesmo acontece quanto fixarmos o número de ativos e aumentamos o $N$ da amostra, exceto na carteira com $1 / d$, que há uma aumento da volatilidade em carteiras com maiores números de ativos. 


\section{Conclusão}

Este trabalho apresenta uma metodologia para construção de conjuntos de incerteza poliedrais data-driven para modelo de distributionally robust optimization de portfólio para um período, tendo como base os retornos observados dos ativos disponíveis. Consideramos diferentes tamanhos de amostras para construção destes conjuntos, de forma que descartamos o dado mais antigo e incluímos na amostra o dado mais recente à cada período $t$ que avançamos. Testamos o modelo sem considerar os custos de transação, volume negociado dos ativos ou quaisquer outras restrições, podendo gerar decisões de alocação completamente diferentes da realidade, tanto em carteiras com tamanhos distintos quanto em diferentes cenários econômicos. Não consideramos o ativo livre de risco como uma opção de investimento, pois queríamos comparar diretamente a estratégia proposta com a carteira igualitária. Também não permitimos venda à descoberto nem alavancagem em relação ao patrimônio para termos um maior controle do risco que buscávamos analisar.

Os resultados empíricos, mostram que no geral, o modelo é uma opção superior ao benchmark, quando consideramos as carteiras com janelas mais longas (amostra $\Theta_{N}$ maior), enquanto as estratégias com janelas curtas performam bem, apenas para carteiras com até 5 ativos, perdendo qualidade em dimensões superiores. Identificamos nos mercados com 5 ativos o melhor desempenho geral do modelo, pois neste cenário, aparentemente atingiu a melhor capacidade de adaptação das diferentes condições de mercado que se realizam, permitindo tirar maior proveito da dinâmica dos preços dos ativos. Em suma, o modelo foi capaz de se proteger nos cenários com maior volatilidade, com isto, quando comparado com os respectivos benchmarks, realizou perdas menores e ganhos maiores. Também reconhecemos um padrão comportamental do modelo quando aumentamos o número de ativos, onde as carteiras com janelas maiores obtiveram um ganho de performance enquanto as com janelas menores perdem significativamente sua qualidade.

Quando aumentamos a janela observada, o modelo passa adotar um padrão mais passivo de alocação, isto é, faz poucas alterações nas alocações, o que geraria um menor impacto no seu desempenho quando incluirmos, na análise, os custos de transação, que pretendemos investigar em um próximo trabalho. 


\section{Referências bibliográficas}

[1] Artzner P., Delbaen F., Eber JM, Heath D. Coerent Measures of Risk. Mathematical Finance (1999), 9(3): 203-228.

[2] Ben-Tal A., Bertsimas D., Brown DB., A soft robust model for otimization under ambiguity. Operations Research (2010) ,58(4): 1220-1234.

[3] Ben-Tal A., Ghaoui El.,Nemirovisky A., Robust Otimization. Princeton University Press (2009).

[4] Ben-Tal A., Nemirovski A. Robust convex optimization. Mathematics of Operations Research (1998), 23(4): 769-805.

[5] Ben-Tal A., Nemirovski A. Robust solutions of uncertain linear programs. Operations Research Letters 25 (1999): 1-13.

[6] Ben-Tal A., Nemirovski A. Robust solutions of Linear Programming problems contaminated with uncertain data. Math Program.,Ser. A 88 (2000): 411-424.

[7] Bernartzi S., Thaler H., Naive Diversification Strategies in Defined Contribution Saving Plans., The American Economic Review (2001), 91(1):79-98.

[8] Bertsimas D., Brown DB., Constructing uncertainty sets for rosbust linear optimization. Operations Reasearch (2009), 56(6): 1483-1495.

[9] Bertsimas D., Sim M. The Price of Robustness. Operations Research (2004), 52(1):35-53.

[10] Bertsimas D., Sim M. Tractable approximations to robust conic optimation problems. Mathematical Programming (2006), 107(1-2):536.

[11] Bertsimas D., Popescu I., Optimal inequalities in probability theory: A convex optimization approach. SIAM J. Optim. (2004), 15(3): 780804.

[12] Calafiore GC., Ghaoui L., On distributionally robust chance constrained linear programs. J. Optim. theory Appl. (2006), 130(1): 1-22. 
[13] Chen W., Sim M., Sun J., Teo C-P., From CVaR to uncertainty set: Implications in joint chance constrained optimization. Operations Research (2010), 58(2):470-485

[14] Cheung SS., So AM-C., Wang K. Linear matrix inequalities with stochastic dependent pertubations and applications to chanceconstrained semidefinide optimization. SIAM J. Optim. (2012), 22(4): 1394-1430.

[15] Delale E., Distributionally robust optimization in context of datadriven problems. Doctoral dissertation (2009), Stanford University, Stanford, CA.

[16] Delale E., Ye Y., Distributionally robust optimization under moments uncertainty with applications to data-driven problems. Operations Research (2010), 58(3): 596-612.

[17] DeMiguel V., Martin-Utrera A., Nogales F.J. Size matters: Optimal calibration of shrinkage estimators for portfolio selection. Journal of Banking and Finance (2013), 37(8): 3018-3034

[18] DeMiguel V., Garlappi L., Uppal, Raman Optimal Versus Naive Diversification: How Inefficient is the 1/N Portfolio Strategy? Oxford University Press, The Review of Finantial Studies (2009) 22(5):1915-1953.

[19] El Ghaoui L., Lebret.H, Robust Solutions to least-Squares Problems with Uncertain Data. SIAM J. Matrix Anal. (1997), 18(4): 1035-1064.

[20] El Ghaoui L., Oustry F., Lebret H. Robust Solution to Uncertain Semidefinite Programs. SIAM J. OPTIM. (1998), 9(1): 33-52.

[21] El Ghaoui L., Oks M., Oustry F., Worse-case value-at-risk and robust portfólio optimization: A conic programming approach. Operations Research (2003), 51(4): 543-556.

[22] Epstein LG. Lectures on Mixing Times. Review of Economic Studies (1999):66, 579-608.

[23] Gilboa I., Schmeider D. Maxmin expected utility with non-unique prior. Journal of Mathematical Economics (1989) 18(2): 141-1

[24] Goh J., Sim M., Distributionally robust optimization and its tractable aproximations. Operations Research (2010), 58(4): 902-917. 
[25] Ishi K. On sharpness od Tchebychevffitype inequalities. Ann. Inst. Statist Math. (1962), 14(1):185-197.

[26] Jagannathan R., Ma T., Risk reduction in large portfolios: Why imposing the wrong constraints helps. Journal of Finance (2003), 58 (5): 1651-1684

[27] Jorion P. Value at Risk: The New chmark for managing finantial risk. McGraw-Hill (2006), ed.3.

[28] Pacote Julia Pro Personal, verão v0.6.3.1, Disponível em: $<$ https://juliacomputing.com/products/juliapro.html>

[29] Kenneth R. Portfolio Industry, (Data library) http://mba.tuck.dartmouth.edu/pages/faculty/ken.french/index.html.

[30] Kuhn D., Wieselman W., Georghiou A., Primal and dual linear decision rules in stochastic and robust optimization. Math. Programming (2011), 130(1): 177-209.

[31] Lagoa CM., Barmish BR., Distributionally robust Monte Carlo simulations: A tutorial survey. Basaña, L., de la Puente, JA. eds. Proc. 15th Internat. Federation Automatic ControlWorld Congress, Vol 15. Elsevier, Amsterdam (1974): 1-12.

[32] Levy H., Levy M., The benefits of differential variance-based constraints in portfolio optimization. European Journal of Operational Research (2014), 234(2): 372-

[33] Markiwitz H., Todd P., Xu, G., Yamana Y. Computation and meansemivariance efficient sets by the critical line algorithm Annals of Operations Research (1993),45(1): 307-3017.

[34] Markowitz, H., Portfolio Selection: Efficient Diversification of Investments., New York, NY, John Wiley \& Sons (1959).

[35] Markowitz H., Portfolio selection. Journal of Finance (1952), 7 (1): 77-91

[36] Merton R.C., On estimating the expected return on the market: An exploratory investigation. Journal of Financial Economics (1980), 8(4): 323-361

[37] Natarajan K., Pachamanova D., Sim M., Constructing risk measures fom uncertainty sets. Operations Research (2009), 57(5): 1129-1141. 
[38] Palczewski A., Palczewski J., Theoretical and empirical estimates of mean variance portfolio sensitivity. European Journal of Operational Research (2014), 234(2): 402-410

[39] Pfug G., Pichler A., Wozabal D., The $1 / \mathrm{N}$ investment strategy is optimal under high model ambiguity. Journal of Banking \& Finance, 36(2): 410-417.

[40] Rockafellar R.T., Uryasev S.P., Optimation fo conditional Value-atRisk. Journal of Risk (2000): vol. 2: 21-41.

[41] Righi M., Ceretta P., Teoria de Medidas de Risco: Uma Revisão Abrangente Revista Brasileira de Finanças (20154), 12(3): 411-464.

[42] Scarf HE. A min-max solution of an inventory problem. Arrow, KJ., Karlin, S., Scarf, HE., eds., Studies in The Mathematical Theory of Inventory and Production Stanford University Press, Stanforf CA (1958):201-209.

[43] Shapiro A., Ahmed S., On a class os minimax stochastic programs. SIAM J. Optim. (2004) 14(4): 1237-1249.

[44] Shapiro A., Kleywegt A., Minimax analysis os stochastic problems. Optim. Methods and Software (2002) 17(3): 523-542.

[45] Shapiro A., On duality theory of conic linear problems. SemiInfinite Programming, Chap. 7(Kluwer Academic Publishers, Dordrecht, the Netherlands) (2001): 135-165.

[46] Site Yahoo! Finance, Dados disponíveis em: $<$ https://finance.yahoo.com/>.

[47] Soyster A.L., Technical Note-Convex Programming with SetInclusive Constraints and Applications to Inexact Linear Programming. Operations Research (1973), 21(5):1154-1157.

[48] Soyster A.L., Technical Note-A Duality Theory for Convex Programming with Set-Inclusive Constraints. Operations Research (1974), 22(4):892-898.

[49] Street A., On the conditional Value-at-Risk probability dependent utility function. Theory and Decision (2010), vol. 68: 49-68.

[50] TLi X., Natarajan K., Teo C-P., Zheng Z., Distribuitionally robust mixed integer linear programs: Persistency models with applications. Eur. J. Oper. Res. (2014), 233(3): 459-473. 
[51] FICO Xpress-Optimizer, Disponível em: <http://www. fico.com/br/products/ficoxpress-optimization>

[52] Xu H., Mannor S., Distributionally robust Markov decision processes. Math. Oper. Res. (2012), 37(2):288-300.

[53] Wolfram Wieselann, Daniel Khun, Melvyn Sim Distributionally Robust Convex Optimization. Operation Research (2014), 62(6):1358-1376.

[54] Za cková J. On minimax solutions of stochastic linear programming problems. Casopis pro pestoáaní matematiky 91(4):423-430

[55] Zymler S., Kuhn D. Rustem B. Distributionally robust joint chance constraint with second-order moments information. Math. Programming (2013), 137(1-2): 167-198. 


\section{Apêndice}

Tabela A.1: Tabelas com o retorno diário médio das carteiras com 2, 5, 10, 17 e 30 ativos.

\section{RETORNO DIÁRIO MÉDIO}

Estratégias 2 ATIVOS 5 ATIVOS 10 ATIVOS 17 ATIVOS 30 ATIVOS

\begin{tabular}{llllll}
\hline Chull $_{5}$ & $0.0375 \%$ & $0.0357 \%$ & $0.0382 \%$ & $0.0304 \%$ & $0.0369 \%$ \\
Chull $_{10}$ & $0.0415 \%$ & $0.0442 \%$ & $0.0316 \%$ & $0.0293 \%$ & $0.0263 \%$ \\
Chull $_{15}$ & $0.0396 \%$ & $0.0465 \%$ & $0.0314 \%$ & $0.0318 \%$ & $0.0249 \%$ \\
Chull $_{30}$ & $0.0370 \%$ & $0.0413 \%$ & $0.0292 \%$ & $0.0282 \%$ & $0.0238 \%$ \\
Chull $_{50}$ & $0.0355 \%$ & $0.0392 \%$ & $0.0314 \%$ & $0.0318 \%$ & $0.0255 \%$ \\
Chull $_{100}$ & $0.0349 \%$ & $0.0373 \%$ & $0.0356 \%$ & $0.0323 \%$ & $0.0242 \%$ \\
Chull $_{252}$ & $0.0345 \%$ & $0.0389 \%$ & $0.0372 \%$ & $0.0328 \%$ & $0.0309 \%$ \\
Chull $_{350}$ & $0.0394 \%$ & $0.0402 \%$ & $0.0399 \%$ & $0.0331 \%$ & $0.0397 \%$ \\
Chull $_{400}$ & $0.0401 \%$ & $0.0407 \%$ & $0.0420 \%$ & $0.0357 \%$ & $0.0457 \%$ \\
Chull $_{450}$ & $0.0380 \%$ & $0.0421 \%$ & $0.0425 \%$ & $0.0339 \%$ & $0.0421 \%$ \\
Chull $_{500}$ & $0.0385 \%$ & $0.0422 \%$ & $0.0440 \%$ & $0.0347 \%$ & $0.0456 \%$ \\
Chull $_{600}$ & $0.0390 \%$ & $0.0404 \%$ & $0.0374 \%$ & $0.0345 \%$ & $0.0451 \%$ \\
\hline Cart. 1/d & $0.0384 \%$ & $0.0369 \%$ & $0.0367 \%$ & $0.0393 \%$ & $0.0404 \%$ \\
\hline
\end{tabular}

Tabela A.2: Tabelas com a proporção de dias em que cada uma das carteiras Chull com 2, 5, 10, 17 e 30 ativos ganhou da carteira 1/d.

PROPORÇÃO DE DIAS QUE GANHOU DA CARTEIRA 1/d Estratégias 2 ATIVOS 5 ATIVOS 10 ATIVOS 17 ATIVOS 30 ATIVOS

\begin{tabular}{llllll}
\hline Chull $_{5}$ & $48.46 \%$ & $48.92 \%$ & $48.38 \%$ & $48.74 \%$ & $48.74 \%$ \\
Chull $_{10}$ & $48.34 \%$ & $48.90 \%$ & $48.56 \%$ & $48.48 \%$ & $48.16 \%$ \\
Chull $_{15}$ & $48.24 \%$ & $48.58 \%$ & $48.52 \%$ & $48.94 \%$ & $48.12 \%$ \\
Chull $_{30}$ & $48.28 \%$ & $48.58 \%$ & $48.46 \%$ & $47.87 \%$ & $47.69 \%$ \\
Chull $_{50}$ & $47.83 \%$ & $48.28 \%$ & $48.22 \%$ & $47.47 \%$ & $47.73 \%$ \\
Chull $_{100}$ & $47.95 \%$ & $48.16 \%$ & $48.36 \%$ & $48.03 \%$ & $47.83 \%$ \\
Chull $_{252}$ & $48.08 \%$ & $48.07 \%$ & $48.38 \%$ & $48.07 \%$ & $48.14 \%$ \\
Chull $_{350}$ & $48.28 \%$ & $48.07 \%$ & $47.99 \%$ & $48.38 \%$ & $48.48 \%$ \\
Chull $_{400}$ & $48.26 \%$ & $48.22 \%$ & $48.24 \%$ & $48.03 \%$ & $48.50 \%$ \\
Chull $_{450}$ & $48.32 \%$ & $48.08 \%$ & $48.50 \%$ & $47.79 \%$ & $48.42 \%$ \\
Chull $_{500}$ & $48.30 \%$ & $48.28 \%$ & $48.70 \%$ & $48.10 \%$ & $48.48 \%$ \\
Chull $_{600}$ & $48.42 \%$ & $48.30 \%$ & $48.26 \%$ & $47.73 \%$ & $48.38 \%$ \\
\hline
\end{tabular}


Tabela A.3: Tabelas de performance das carteiras elaboradas com o DPOM com 5 e 10 ativos.

\section{CARTEIRA COM 5 ATIVOS}

\begin{tabular}{lcccccc} 
Estratégia & $\mathbf{R}_{\mathbf{T}}$ & $\mathbf{r}_{\mathbf{p}}$ & $\mathbf{\sigma}_{\mathbf{p}}$ & $\mathbf{I S}_{\mathbf{p}}$ & $\mathbf{M D D}_{\mathbf{T}}$ & $\begin{array}{c}\text { Tempo } \\
\text { Computacional }\end{array}$ \\
\hline Chull $_{5}$ & $340.49 \%$ & $7.70 \%$ & $16.01 \%$ & 0.39 & $-45.27 \%$ & 0.007 \\
Chull $_{10}$ & $605.62 \%$ & $10.26 \%$ & $15.40 \%$ & 0.57 & $-38.66 \%$ & 0.011 \\
Chull $_{15}$ & $696.83 \%$ & $10.93 \%$ & $13.88 \%$ & 0.68 & $-34.07 \%$ & 0.001 \\
Chull $_{30}$ & $517.68 \%$ & $9.53 \%$ & $15.30 \%$ & 0.53 & $-39.28 \%$ & 0.039 \\
Chull $_{50}$ & $454.53 \%$ & $8.94 \%$ & $16.29 \%$ & 0.46 & $-43.74 \%$ & 0.082 \\
Chull $_{100}$ & $405.34 \%$ & $8.44 \%$ & $15.76 \%$ & 0.44 & $-43.60 \%$ & 0.340 \\
Chull $_{252}$ & $444.28 \%$ & $8.84 \%$ & $15.48 \%$ & 0.48 & $-40.45 \%$ & 1.358 \\
Chull $_{350}$ & $483.02 \%$ & $9.22 \%$ & $14.23 \%$ & 0.55 & $-38.55 \%$ & 2.603 \\
Chull $_{400}$ & $498.64 \%$ & $9.36 \%$ & $14.82 \%$ & 0.54 & $-37.34 \%$ & 3.505 \\
Chull $_{450}$ & $539.45 \%$ & $9.72 \%$ & $14.77 \%$ & 0.56 & $-37.34 \%$ & 4.719 \\
Chull $_{500}$ & $543.60 \%$ & $9.76 \%$ & $12.93 \%$ & 0.64 & $-39.56 \%$ & 6.026 \\
Chull $_{600}$ & $485.99 \%$ & $9.24 \%$ & $14.04 \%$ & 0.56 & $-37.34 \%$ & 11.230 \\
\hline Cart. 1/d & $352.00 \%$ & $7.83 \%$ & $16.07 \%$ & 0.40 & $-51.24 \%$ & - \\
\hline
\end{tabular}

\section{CARTEIRA COM 10 ATIVOS}

\begin{tabular}{lcccccc} 
Estratégia & $\mathbf{R}_{\mathbf{T}}$ & $\mathbf{r}_{\mathbf{p}}$ & $\boldsymbol{\sigma}_{\mathbf{p}}$ & $\mathbf{I S}_{\mathbf{p}}$ & $\mathbf{M D D}_{\mathbf{T}}$ & $\begin{array}{c}\text { Tempo } \\
\text { Computacional }\end{array}$ \\
\hline Chull $_{5}$ & $404.55 \%$ & $8.43 \%$ & $19.77 \%$ & 0.35 & $-56.30 \%$ & 0.006 \\
Chull $_{10}$ & $283.11 \%$ & $6.95 \%$ & $17.57 \%$ & 0.31 & $-47.32 \%$ & 0.011 \\
Chull $_{15}$ & $281.60 \%$ & $6.93 \%$ & $17.03 \%$ & 0.32 & $-47.05 \%$ & 0.016 \\
Chull $_{30}$ & $245.79 \%$ & $6.40 \%$ & $14.89 \%$ & 0.33 & $-47.30 \%$ & 0.038 \\
Chull $_{50}$ & $288.81 \%$ & $7.03 \%$ & $16.95 \%$ & 0.33 & $-53.27 \%$ & 0.080 \\
Chull $_{100}$ & $381.49 \%$ & $8.18 \%$ & $15.93 \%$ & 0.42 & $-48.26 \%$ & 0.282 \\
Chull $_{252}$ & $418.71 \%$ & $8.58 \%$ & $14.45 \%$ & 0.49 & $-44.07 \%$ & 1.170 \\
Chull $_{350}$ & $492.94 \%$ & $9.31 \%$ & $12.93 \%$ & 0.61 & $-39.56 \%$ & 2.305 \\
Chull $_{400}$ & $557.90 \%$ & $9.88 \%$ & $14.82 \%$ & 0.57 & $-42.18 \%$ & 2.675 \\
Chull $_{450}$ & $574.01 \%$ & $10.01 \%$ & $12.93 \%$ & 0.66 & $-39.56 \%$ & 4.115 \\
Chull $_{500}$ & $623.01 \%$ & $10.40 \%$ & $12.93 \%$ & 0.69 & $-39.56 \%$ & 4.702 \\
Chull $_{600}$ & $416.70 \%$ & $8.56 \%$ & $14.53 \%$ & 0.49 & $-42.18 \%$ & 7.936 \\
\hline Cart. 1/d & $352.32 \%$ & $7.84 \%$ & $16.25 \%$ & 0.39 & $-52.49 \%$ & - \\
\hline
\end{tabular}


Tabela A.4: Tabelas de performance das carteiras elaboradas com o DPOM com 17 e 30 ativos.

\section{CARTEIRA COM 17 ATIVOS}

\begin{tabular}{lcccccc} 
Estratégia & $\mathbf{R}_{\mathbf{T}}$ & $\mathbf{r}_{\mathbf{p}}$ & $\mathbf{\sigma}_{\mathbf{p}}$ & $\mathbf{I S}_{\mathbf{p}}$ & $\mathbf{M D D}_{\mathbf{T}}$ & $\begin{array}{c}\text { Tempo } \\
\text { Computacional }\end{array}$ \\
\hline Chull $_{5}$ & $226.63 \%$ & $6.10 \%$ & $19.23 \%$ & 0.24 & $-56.91 \%$ & 0.006 \\
Chull $_{10}$ & $239.88 \%$ & $6.31 \%$ & $16.00 \%$ & 0.30 & $-51.76 \%$ & 0.011 \\
Chull $_{15}$ & $290.44 \%$ & $7.05 \%$ & $14.78 \%$ & 0.38 & $-43.48 \%$ & 0.018 \\
Chull $_{30}$ & $229.81 \%$ & $6.15 \%$ & $13.57 \%$ & 0.35 & $-48.16 \%$ & 0.044 \\
Chull $_{50}$ & $296.82 \%$ & $7.13 \%$ & $14.64 \%$ & 0.39 & $-48.71 \%$ & 0.106 \\
Chull $_{100}$ & $307.45 \%$ & $7.28 \%$ & $13.30 \%$ & 0.44 & $-42.42 \%$ & 0.289 \\
Chull $_{252}$ & $312.45 \%$ & $7.34 \%$ & $14.73 \%$ & 0.40 & $-49.81 \%$ & 1.398 \\
Chull $_{350}$ & $318.85 \%$ & $7.42 \%$ & $14.81 \%$ & 0.40 & $-50.79 \%$ & 3.682 \\
Chull $_{400}$ & $377.59 \%$ & $8.13 \%$ & $14.67 \%$ & 0.46 & $-50.25 \%$ & 3.319 \\
Chull $_{450}$ & $335.74 \%$ & $7.64 \%$ & $14.37 \%$ & 0.43 & $-50.12 \%$ & 7.491 \\
Chull $_{500}$ & $352.94 \%$ & $7.85 \%$ & $14.32 \%$ & 0.45 & $-49.69 \%$ & 8.782 \\
Chull $_{600}$ & $349.34 \%$ & $7.80 \%$ & $14.75 \%$ & 0.43 & $-49.58 \%$ & 11.728 \\
\hline Cart. 1/d & $387.24 \%$ & $8.24 \%$ & $17.36 \%$ & 0.39 & $-53.93 \%$ & - \\
\hline \multicolumn{7}{c}{}
\end{tabular}

\section{CARTEIRA COM 30 ATIVOS}

\begin{tabular}{lcccccc} 
Estratégia & $\mathbf{R}_{\mathbf{T}}$ & $\mathbf{r}_{\mathbf{p}}$ & $\boldsymbol{\sigma}_{\mathbf{p}}$ & $\mathbf{I S}_{\mathbf{p}}$ & $\mathbf{M D D}_{\mathbf{T}}$ & $\begin{array}{c}\text { Tempo } \\
\text { Computacional }\end{array}$ \\
\hline Chull $_{5}$ & $332.09 \%$ & $7.59 \%$ & $19.64 \%$ & 0.31 & $-49.92 \%$ & 0.006 \\
Chull $_{10}$ & $189.48 \%$ & $5.46 \%$ & $18.05 \%$ & 0.22 & $-59.23 \%$ & 0.013 \\
Chull $_{15}$ & $175.82 \%$ & $5.20 \%$ & $17.85 \%$ & 0.21 & $-60.90 \%$ & 0.021 \\
Chull $_{30}$ & $165.23 \%$ & $5.00 \%$ & $16.40 \%$ & 0.22 & $-48.62 \%$ & 0.059 \\
Chull $_{50}$ & $190.04 \%$ & $5.47 \%$ & $15.76 \%$ & 0.26 & $-53.15 \%$ & 0.151 \\
Chull $_{100}$ & $172.33 \%$ & $5.14 \%$ & $13.49 \%$ & 0.27 & $-40.63 \%$ & 0.450 \\
Chull $_{252}$ & $281.02 \%$ & $6.92 \%$ & $12.93 \%$ & 0.42 & $-39.56 \%$ & 1.127 \\
Chull $_{350}$ & $489.98 \%$ & $9.28 \%$ & $14.77 \%$ & 0.53 & $-40.92 \%$ & 2.820 \\
Chull $_{400}$ & $696.65 \%$ & $10.93 \%$ & $15.19 \%$ & 0.63 & $-39.33 \%$ & 5.071 \\
Chull $_{450}$ & $565.87 \%$ & $9.94 \%$ & $14.87 \%$ & 0.57 & $-38.67 \%$ & 8.619 \\
Chull $_{500}$ & $689.93 \%$ & $10.89 \%$ & $14.69 \%$ & 0.64 & $-38.67 \%$ & 11.851 \\
Chull $_{600}$ & $667.30 \%$ & $10.73 \%$ & $15.08 \%$ & 0.62 & $-38.67 \%$ & 11.851 \\
\hline Cart. 1/d & $423.70 \%$ & $8.63 \%$ & $17.83 \%$ & 0.40 & $-57.09 \%$ & - \\
\hline
\end{tabular}

